\title{
Constructing Out-of-the-Money Longevity Hedges Using Parametric Mortality Indexes
}

\author{
Johnny Siu-Hang Li; Jackie Li† Uditha Balasooriya
}

\begin{abstract}
Proposed by Chan et al. (2014), parametric mortality indexes (i.e., indexes created using the time-varying parameters in a suitable stochastic mortality model) can be used to develop tradable mortality-linked derivatives such as K-forwards. Compared to existing indexes such as the LLMA's LifeMetrics, parametric mortality indexes are richer in information content, allowing the market to better concentrate liquidity. In this paper, we further study this concept in several aspects. First, we consider options written on parametric mortality indexes. Such options enable hedgers to create out-of-the-money longevity hedges, which, compared to at-the-money-hedges created with q-/K-forwards, may better meet hedgers' need for protection against downside risk. Second, using the properties of the time-series processes for the parametric mortality indexes, we derive analytical risk-neutral pricing formulas for K-forwards and options. In addition to convenience, the analytical pricing formulas remove the need for computationally intensive nested simulations that are entailed in, for example, the calculation of the hedging instruments' values when a dynamic hedge is adjusted. Finally, we construct static and dynamic Greek hedging strategies using K-forwards and options, and demonstrate empirically the conditions under which an out-of-the-money hedge is more economically justifiable than an at-the-money one.
\end{abstract}

Keywords: K-forwards; K-options; Longevity Greeks; Risk-neutral pricing; The CBD mortality indexes; Value-at-Risk.

\section{Introduction}

The notion of parametric mortality indexes was originally proposed by Chan et al. (2014). Parametric mortality indexes are mortality indexes that are created using the time-varying parameters

\footnotetext{
*Department of Economics, The University of Melbourne, Australia; Department of Statistics and Actuarial Science, University of Waterloo, Canada

${ }^{\dagger}$ Department of Applied Finance and Actuarial Studies, Macquarie University, Australia

${ }^{\ddagger}$ Insurance Risk and Finance Research Centre, Division of Banking and Finance, Nanyang Business School, Nanyang Technological University, Singapore

${ }^{\S}$ Department of Statistics and Actuarial Science, University of Waterloo, Canada
} 
in an appropriately chosen stochastic mortality model. They summarize how the mortality curve of a certain population evolves over time, and the random deviations from their expected trajectories reflect the level of longevity risk that the population is subject to. To fully utilize the potential of parametric mortality indexes, Chan et al. (2014) further proposed a conceptual security called K-forward, which refers to a zero-coupon swap that is written on a specific parametric mortality index. Through K-forwards, pension plan providers can hedge their longevity risk exposures, while capital market investors can tap into the longevity asset class which offers them risk premium and diversification benefits.

Compared to non-parametric mortality indexes such as the LLMA's LifeMetrics and Deutsche Börse's Xpect Cohort Indexes, parametric mortality indexes are advantageous of being richer in information content, so that non-parallel shifts in the underlying mortality curve over time can be captured with only a few indexes. This property enables the market to better concentrate liquidity, fostering the popularity of less costly index-based longevity hedging solutions. Further, depending on the model from which they are created, parametric mortality indexes are often highly interpretable. For instance, the two parametric mortality indexes (the CBD mortality indexes) recommended by Chan et al. (2014) can be understood as the level and gradient of the (logit-transformed) underlying mortality curve, respectively. The ease of interpretation makes Kforwards and other securities written on parametric mortality indexes more marketable to capital market investors.

Since proposed by Chan et al. (2014), parametric mortality indexes and K-forwards have been further studied by various researchers. Tan et al. (2014) examined how a static K-forward hedge may be calibrated with a 'duration-matching' approach, and demonstrated that a K-forward hedge has a potential to outperform a q-forward hedge in terms of the number of hedging instruments required. Hao et al. (2017) studied the counterparty credit risk associated with K-forwards. In particular, they derived the risk-neutral default probability (from the hedge provider's perspective), and calculated the credit value adjustment in different circumstances. Wei (2017) modelled the CBD mortality indexes with the LLCBD model proposed by Liu and Li (2017), and performed a further study on the counterparty credit risk concerning K-forwards. Biffis et al. (2017) introduced a security that is written on the population-specific time-varying parameters in the augmented common factor model (Li and Lee, 2005). Although they name their security slightly differently ('k-forward' instead of 'K-forward'), the spirit behind (i.e., writing securities on the time-varying parameters in a stochastic mortality model) is essentially the same.

In this paper, we revisit the concepts of parametric mortality indexes and K-forwards, with a focus on three specific objectives. First, we explore security structures other than a zero coupon swap, and examine how the alternative security structures may benefit the hedger. Second, we study risk-neutral valuation of K-forwards and other securities written on parametric mortality indexes. This objective distinguishes our paper from the previous work on parametric mortality indexes by Biffis et al. (2017) and Tan et al. (2014), who ignored the cost of hedging completely. Third, we consider dynamic hedging with K-forwards and options, extending the work Tan et 
al. (2014) who focused on static hedging and used forward contracts only. The construction of parametric mortality indexes, which was studied previously by Chan et al.(2014) and Tan et al. (2015), is beyond the scope of this paper. We choose to focus on the CBD mortality indexes, which satisfy the three key criteria set out by Chan et al. (2014).

In terms of security structures, we particularly focus on options written on parametric mortality indexes (thereafter referred to as K-options). Longevity securities with option-like payoffs were discussed in the early work of Blake et al. (2006) and Cairns et al. (2006a, 2008), and have received considerable attention recently (e.g., Cairns and Boukfaoui, 2017). As Michaelson and Mulholland (2014) pointed out, longevity securities featuring option-like payoffs enable hedgers to create out-of-the-money hedges against extreme downside longevity outcomes, while retaining the potential upside gain. Our aim is to investigate the feasibility of building out-of-the-money longevity hedges using K-options, and examine how such hedges perform compared to their atthe-money counterparts in different circumstances. A similar study concerning payoff structures of longevity securities was conducted by Biffis and Blake (2010), who concluded that in some cases it is optimal to transfer longevity risk using a call (put) option on mortality (survival) rates. It should be noted that their conclusion is based on the assumption of a Walrasian economy, whereas in our investigation we assume that hedgers (buyers of K-options) are price takers. Another noteworthy distinction is that the liability being hedged in our set-up is apparently more realistic than the 'annuity-like' liability that Biffis and Blake (2010) considered.

In terms of pricing, we strive to derive analytical (risk-neutral) pricing formulas for K-forwards and options. When considering derivatives on mortality and survival rates (e.g., q-forwards and S-forwards), analytical pricing formulas are available only under rather restrictive set-ups (see, e.g., Dahl, 2004; Bauer et al., 2008, 2010). ${ }^{1}$ In more general situations, prices of derivatives on mortality and survival rates have to be estimated using simulations (see, e.g., Boyer and Stentoft, 2013; Li, 2010; Li and Ng, 2011) or closed-form expressions that involve a certain extent of approximations (see, e.g., Wang and Yang, 2013). Nevertheless, thanks to the fact that Kforwards and options are written directly on the time-varying parameters which are assumed follow some tractable time-series processes, analytical pricing formulas for there securities can be derived readily using the structural and statistical properties of the risk-adjusted version of the assumed processes. On the basis of the risk-neutral Cairns-Blake-Dowd model specified by Cairns et al. (2006b), we derive analytical pricing formulas for K-forwards and options written on the CBD mortality indexes. The resulting formulas are intuitive, and satisfy the put-call parity relationship as the Black-Scholes formula for equity options does.

In terms of hedging, we consider both static and dynamic hedging using the CBD mortality indexes. Our work on dynamic hedging draws from the previous contributions of Cairns (2011) and Zhou and Li (2017a,b), who avoided the need for the computationally demanding nested simulations entailed at each time point when the hedge is rebalanced using the 'approximation

\footnotetext{
${ }^{1}$ For instance, the assumed model in the set-up of Bauer et al. (2010) may yield negative forces of mortality.
} 
of survival function' method. One drawback of this method, as Cairns (2011, p.449) mentioned, is that it does not work satisfactorily when the hedging instruments have a non-linear payoff structure. However, this drawback does not apply to our K-options, as their prices and Greek letters can be calculated analytically (without any approximation). We consider both cash flow hedges (which focus on the variability of the cash flows arising from the hedged position) and value hedges (which focus on the variability of the values of hedged position at a certain future time point). For both, we measure hedge effectiveness in terms of Value-at-Risk, which takes the cost of hedging into consideration.

We provide real data illustrations of the synthesis of our theoretical contributions. Through a range of sensitivity tests, we arrive at several interesting conclusions. For instance, a K-put hedge is more likely to yield a lower Value-at-Risk than a K-forward hedge when the market prices of risk are high and/or the times-to-maturity of the hedging instruments are long. Another example is that a value hedge is highly robust to changes in the market prices of risk, the risk-free interest rate, and the times-to-maturity of the hedging instruments.

The rest of this paper is structured as follows. Section 2 reviews the CBD mortality indexes and their merits. Section 3 defines the risk-neutral process for the CBD mortality indexes. Section 4 presents the derivation of the exact analytical risk-neutral pricing formulas for K-forwards and options. Section 5 specifies the liability being hedged and describes the approximation method for valuing it. Section 6 details our theoretical work on static and dynamic delta hedging using K-forwards and options. Section 7 is devoted to the real data illustrations and sensitivity tests. Finally, Section 8 concludes the paper with a few suggestions for future research.

\section{The CBD Mortality Indexes: A Review}

\subsection{Definitions}

The CBD mortality indexes are developed from the original version of the Cairns-Blake-Dowd model (Cairns et al., 2006b), which can be expressed as

$$
\ln \left(\frac{q_{x, t}}{1-q_{x, t}}\right)=\kappa_{t}^{(1)}+\kappa_{t}^{(2)}(x-\bar{x})
$$

where

- $q_{x, t}$ is the probability that an individual aged $x$ at time $t$ will die between $t$ and $t+1$;

- $\bar{x}$ is the mean age over the sample age range; and

- $\kappa_{t}^{(1)}$ and $\kappa_{t}^{(2)}$ are time-varying parameters.

The time- $t$ values of the first and second CBD mortality indexes are defined as $\kappa_{t}^{(1)}$ and $\kappa_{t}^{(2)}$, respectively. 


\subsection{Interpreting the Indexes}

On the basis of the model structure specified in equation (1), the two CBD mortality indexes can be interpreted easily as follows:

- The first index $\left(\kappa_{t}^{(1)}\right)$ represents the level of the logit-transformed mortality curve (the curve of $q_{x, t}$ in year $\left.t\right) .^{2}$ A reduction in $\kappa_{t}^{(1)}$ implies an overall reduction in mortality, affecting the death probabilities (in logit scale) at all ages by the same amount.

- The second index $\left(\kappa_{t}^{(2)}\right)$ represents the gradient of the logit-transformed mortality curve. A change in $\kappa_{t}^{(2)}$ reflects a change in the distribution of mortality improvement across ages. In particular, an increase in $\kappa_{t}^{(2)}$ means that mortality (in logit scale) at younger ages (below the mean age $\bar{x}$ ) improves more rapidly than at older ages (above the mean age $\bar{x}$ ).

Chan et al. (2014) further defined K1 and K2 risks as the risks surrounding the future values of $\kappa_{t}^{(1)}$ and $\kappa_{t}^{(2)}$, respectively. They noted that pension plans and life insurers are subject to very different profiles of $\mathrm{K} 1$ and $\mathrm{K} 2$ risks.

For closed pension plans, financial obligations are positively related to future mortality improvement at old ages. Therefore, the worst situation for them occurs when (i) the overall mortality improvement is faster than expected (which happens when the first CBD index is lower than expected) and (ii) mortality improvement is more concentrated at older ages (which happens when the second CBD index is lower than expected). It follows that they are subject to a downside $\mathrm{K} 1$ risk and a downside $\mathrm{K} 2$ risk.

For insurance companies with a concentration on term-life business, of which customers are typically young, financial obligations are negatively related to future mortality improvement at young ages. Hence, the worst outcome for them occurs when (i) the overall mortality improvement is slower than expected (which happens when the first CBD index is higher than expected) and (ii) mortality improvement is more concentrated at younger ages (which happens when the second CBD index is lower than expected). It follows that they are subject to an upside K1 risk and a downside $\mathrm{K} 2$ risk.

The above descriptions are summarized in Figure 1. This diagram aids us in determining the appropriate positions of the various hedging instruments that are introduced in Section 3.

\subsection{Properties of the Indexes}

As Chan et al. (2014) mentioned, the CBD mortality indexes possess the following three desirable properties:

1. The indexes are small in dimension, but are able to represent the age-pattern of mortality improvement.

\footnotetext{
${ }^{2}$ The logit transformation of a real number $w$ is given by $\ln (w /(1-w))$.
} 


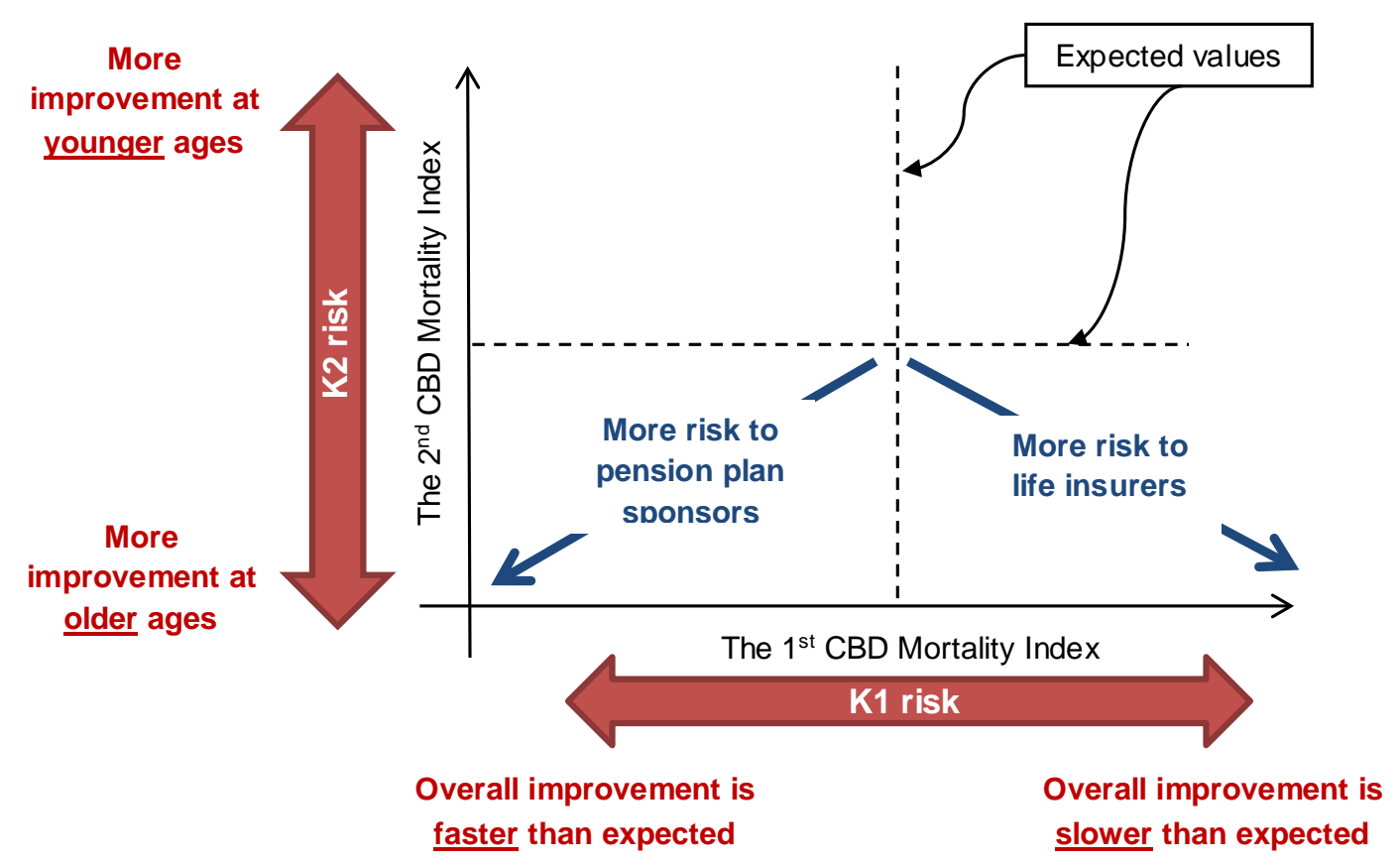

Figure 1: A graphical illustration of $\mathrm{K} 1$ and $\mathrm{K} 2$ risks.

As demonstrated in Figure 2, the two CBD mortality indexes can represent different patterns of the underlying mortality curve. To achieve this ability with non-parametric indexes such as the LLMA's LifeMetric, a much larger number of indexes would be needed. This property enables effective hedging of longevity risk (which arises from random non-parallel shifts in the underlying mortality curve), while allowing the market to concentrate liquidity on a small number of indexes.

2. The indexes are interpretable.

As discussed in the previous sub-section, the two CBD mortality indexes are readily interpretable. The impact of changes in each CBD mortality index on closed pension plans and life insurers can also be clearly understood. This property facilitates marketability of the indexes to both hedgers and capital market investors.

3. The model from which the indexes are derived posseeses the new-data-invariant property.

The new-data-invariant property refers to the situation that when an additional year of data becomes available and the model from which the parametric mortality indexes are derived is updated accordingly, the indexes in previous years would not be affected. This property is crucial, because it would be impossible to track an index if its historical values are revised from time to time. Due to its 'separable' likelihood function and the fact that it is free of any identifiability constraints, the original Cairns-Blake-Dowd model (from which CBD mortality indexes are derived) satisfies the new-data-invariant property (see Chan et 
al., 2014). Note that among the six stochastic mortality models considered by Dowd et al. (2010), the original Cairns-Blake-Dowd model is the only one that satisfies the new-datainvariant property.

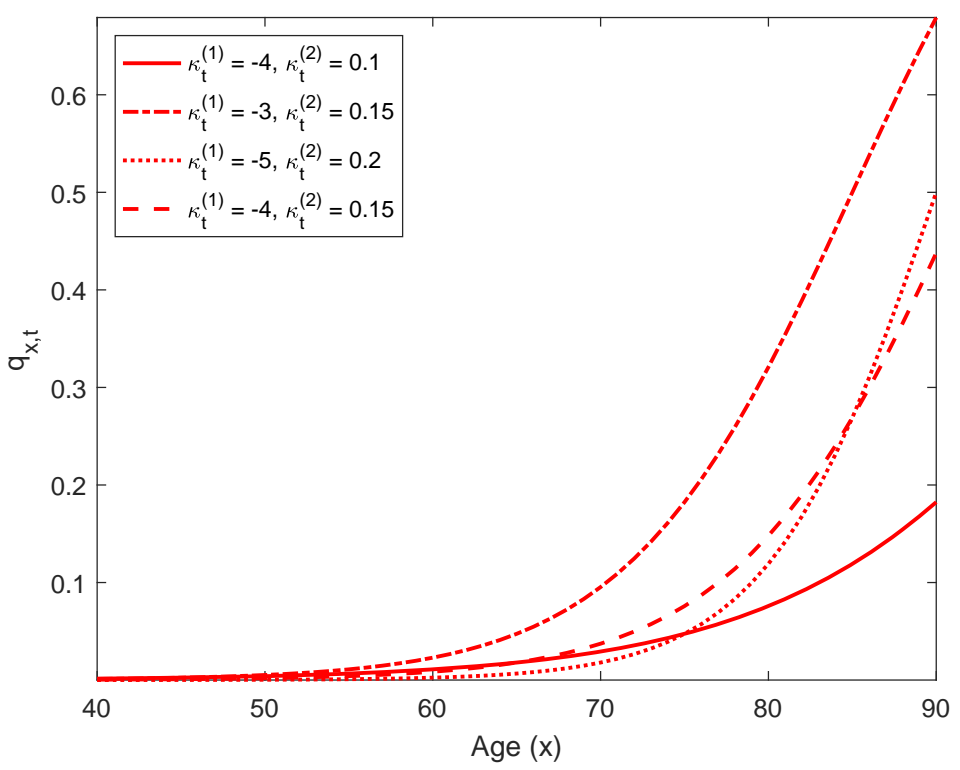

Figure 2: Curves of $q_{x, t}$ for different pairs of CBD mortality indexes at time $t$. The value of $\bar{x}$ in the model is 65 .

\section{The Risk-Neutral Cairns-Blake-Dowd Model}

Under both the real-world and risk-neutral probability measures, the Cairns-Blake-Dowd model links $q_{x, t}$ to the two time-varying parameters $\kappa_{t}^{(1)}$ and $\kappa_{t}^{(2)}$ in exactly the same manner (through equation (1)). However, the stochastic processes driving the dynamics of $\kappa_{t}^{(1)}$ and $\kappa_{t}^{(2)}$ under the two probability measures are different.

Following Cairns et al. (2006b), we assume that under the real-world probability measure, $\kappa_{t}^{(1)}$ and $\kappa_{t}^{(2)}$ follow a bivariate random walk with a constant drift vector:

$$
\boldsymbol{\kappa}_{t}=\boldsymbol{\mu}+\boldsymbol{\kappa}_{t-1}+\mathbf{A} \boldsymbol{z}_{t}
$$

where $\boldsymbol{\kappa}_{t}=\left(\kappa_{t}^{(1)}, \kappa_{t}^{(2)}\right)^{\prime}$ represents the vector of the time-varying parameters, $\boldsymbol{\mu}=\left(\mu^{(1)}, \mu^{(2)}\right)^{\prime}$ is the constant drift vector, $\mathbf{A}$ is a 2-by-2 upper-triangular matrix, and $\boldsymbol{z}_{t}=\left(z_{t}^{(1)}, z_{t}^{(2)}\right)^{\prime}$ is a vector of two uncorrelated standard normal random variables under the real-world probability measure. It is further assumed that $\boldsymbol{z}_{s}$ and $\boldsymbol{z}_{u}$ are uncorrelated if $s \neq u$.

Following Cairns et al. (2006b), we further assume that the risk-neutral dynamics of $\kappa_{t}^{(1)}$ and 
$\kappa_{t}^{(2)}$ are driven by

$$
\boldsymbol{\kappa}_{t}=\boldsymbol{\mu}+\boldsymbol{\kappa}_{t-1}+\mathbf{A}\left(\tilde{z}_{t}-\boldsymbol{\lambda}\right)
$$

where $\tilde{z}_{t}=\left(\tilde{z}_{t}^{(1)}, \tilde{z}_{t}^{(2)}\right)^{\prime}$ is a vector of two uncorrelated standard normal random variables (both of which possess no serial correlation) under the risk-neutral probability measure, $\boldsymbol{\lambda}=\left(\lambda^{(1)}, \lambda^{(2)}\right)^{\prime}$ represents the vector of market prices of risk. We can understand $\lambda^{(1)}$ and $\lambda^{(2)}$ as the market prices of $\mathrm{K} 1$ and $\mathrm{K} 2$ risks, respectively. Rearranging the equation above, we get

$$
\boldsymbol{\kappa}_{t}=\tilde{\boldsymbol{\mu}}+\boldsymbol{\kappa}_{t-1}+\mathbf{A} \tilde{\boldsymbol{z}}_{t}
$$

where $\tilde{\boldsymbol{\mu}}=\boldsymbol{\mu}-\mathbf{A} \boldsymbol{\lambda}$, which says that under the risk-neutral probability measure, $\kappa_{t}^{(1)}$ and $\kappa_{t}^{(2)}$ also follow a random walk but with a constant drift vector that is different from that under the realworld probability measure. It is interesting to note that the relationship between the real-world process (equation (2)) and the risk-neutral process (equation (3)) we consider is consistent with that obtained using the Esscher transform (see Hunt and Blake, 2015).

The market prices of risk are calibrated to market information, for example, market prices of individual life annuities. The calibration work is beyond the scope of this paper. In our baseline calculations, we set $\lambda^{(1)}=\lambda^{(2)}=0.175$. This collection of market prices of risk was obtained by Cairns et al. (2006b) using the market price of the longevity bond jointly announced by BNP Paribas and the European Investment Bank in 2004. ${ }^{3}$ Interested readers are referred to Hunt and Blake (2015; Section 3.2) for a detailed discussion on how the calibration work may be performed in practice.

Finally, it follows from equation (3) that given information up to and including time $t, \kappa_{T}^{(i)}$ for $T>t$ under the risk-neutral probability measure can be expressed as

$$
\kappa_{T}^{(i)}=\kappa_{t}^{(i)}+(T-t) \tilde{\mu}^{(i)}+\sum_{k=1}^{T-t} \tilde{\epsilon}_{t+k}^{(i)}, \quad i=1,2
$$

where $\tilde{\epsilon}_{t+k}^{(1)}=a_{1,1} \tilde{z}_{t+k}^{(1)}+a_{1,2} \tilde{z}_{t+k}^{(2)}$ and $\tilde{\epsilon}_{t+k}^{(2)}=a_{2,2} \tilde{z}_{t+k}^{(2)}$, with $a_{i, j}$ being the $(i, j)$ th element in $\mathbf{A}$. Consequently, given information up to and including time $t, \kappa_{T}^{(i)}$ for $T>t$ under the risk-neutral probability measure is normally distributed with a mean of

$$
\mathrm{E}_{t}^{(Q)}\left[\kappa_{T}^{(i)}\right]=\kappa_{t}^{(i)}+(T-t) \tilde{\mu}^{(i)}, \quad i=1,2,
$$

and a variance of

$$
\operatorname{Var}_{t}^{(Q)}\left[\kappa_{T}^{(i)}\right]= \begin{cases}(T-t)\left(a_{1,1}^{2}+a_{1,2}^{2}\right), & i=1 \\ (T-t) a_{2,2}^{2}, & i=2\end{cases}
$$

\footnotetext{
${ }^{3}$ We fully acknowledge that the calibrated market prices of risk are subject to some limitations: (1) the longevity bond was not actually traded, so that its announced price may not truly reflect the market participants' aversion to longevity risk; (2) the bond was announced more than 10 years ago, so the information contained in its announced price may not be up to date. To mitigate these limitations, we sensitivity test (in Section 7.3) the hedging results using a wide range of market prices of risk, including the extreme case when $\lambda^{(1)}=\lambda^{(2)}=0$.
} 
where $\mathrm{E}_{t}^{(Q)}[\cdot]$ and $\operatorname{Var}_{t}^{(Q)}[\cdot]$ represent the risk-neutral expectation and variance given information up to and including time $t$, respectively. The pricing formulas presented in the next section draw heavily from equations (5) and (6).

\section{The Hedging Instruments}

In this section, we define three securities (K-forward, K-call and K-put) that are written on the CBD mortality indexes, and present the exact analytical pricing formulas for the three securities on the basis of the previously described risk-neutral process. Throughout the rest of the paper, we refer a K-forward written on the first (second) CBD mortality index to as a K1-forward (K2forward). Similar nomenclature applies to K-calls and K-puts.

In preparation for hedging, we also derive the longevity delta and gamma for each of the three securities. As in the previous work of Cairns (2011) and Zhou and Li (2017a,b), the longevity deltas and gammas of a mortality-linked security are defined as the first and second partial derivatives of its value with respect to the most recently realized period effects (the CBD mortality indexes), respectively.

In the end of this section, we study the put-call parity for the securities written on the CBD mortality indexes, as well as how the properties of the securities may change with the level of moneyness.

\subsection{K-forward}

A K-forward is a zero-coupon swap that exchanges on the maturity date a fixed amount for a random amount that is proportional to a CBD mortality index realized at some future time. Consider a K-forward written on the $i$ th CBD mortality index. Suppose that the K-forward is issued at time $t_{0}$ and matures at time $T$ (where $T>t_{0}$ ). From the perspective of the fixed rate receiver, the payoff of this K-forward at maturity is

$$
\mathcal{F}^{(i)}(T, K)=K-\kappa_{T}^{(i)}
$$

per $\$ 1$ notional, where $K$ represents the fixed leg that is predetermined when the K-forward is issued. ${ }^{4}$

The value of this $\mathrm{K}$-forward at time $t$ for $t_{0} \leq t<T$ is the risk-neutral expectation of its payoff $\mathcal{F}^{(i)}(T, K)$, discounted at the risk-free interest rate $r_{f}$, given information up to and including time $t$; that is,

$$
\begin{aligned}
F_{t}^{(i)}(T, K) & =\mathrm{E}_{t}^{(Q)}\left[\left(1+r_{f}\right)^{-(T-t)} \mathcal{F}^{(i)}(T, K)\right] \\
& =\left(1+r_{f}\right)^{-(T-t)}\left(K-\mathrm{E}_{t}^{(Q)}\left[\kappa_{T}^{(i)}\right]\right),
\end{aligned}
$$

\footnotetext{
${ }^{4}$ For simplicity, we ignore the potential lag in the availability of the index data. This assumption is made throughout the rest of the paper.
} 
where $\mathrm{E}_{t}^{(Q)}\left[\kappa_{T}^{(i)}\right]$ is given by equation (5) according to the model assumptions made.

The time- $t$ values of the longevity delta and gamma for this K-forward are the first and second order partial derivatives of $F_{t}^{(i)}(T, K)$ with respect to $\kappa_{t}^{(i)}$, respectively; that is,

$$
\Delta_{t}^{(i, F)}(T, K)=\frac{\partial}{\partial \kappa_{t}^{(i)}} F_{t}^{(i)}(T, K)=-(1+r)^{-(T-t)}
$$

and

$$
\Gamma_{t}^{(i, F)}(T, K)=\frac{\partial}{\partial \kappa_{t}^{(i)}} \Delta_{t}^{(i, F)}(T, K)=0
$$

for $t_{0} \leq t<T$. It is not surprising that $\Gamma_{t}^{(i, F)}(T, K)=0$ for all $t_{0} \leq t<T$ as the payoff is a perfectly linear function of $\kappa_{T}^{(i)}$.

In practice, a K-forward may be constructed in such a way that no cash flow exchanged hands at time $t_{0}$ when it is issued. To achieve this, we require $F_{t_{0}}^{(i)}(T, K)=0$, which in turn means that the fixed leg $K$ should be set to $\mathrm{E}_{t_{0}}^{(Q)}\left[\kappa_{T}^{(i)}\right]$.

As discussed in Section 2.2, pension plan sponsors are subject to downside K1 and K2 risks. Therefore, to hedge their longevity risk exposures, they may participate in K1- and K2-forwards as a fixed-rate receiver, so that they will receive a net payment from the counterparty to defray their increased pension obligations when the CBD mortality indexes turn out to be too low. On the other hand, life insurers, who are typically subject to an upside K1 risk and a downside K2 risk, may hedge their risk exposures by participating as a fixed-rate payer in a K1-forward and a fixed-rate receiver in a $\mathrm{K} 2$-forward.

\subsection{K-call}

Consider a (European) K-call on the $i$ th CBD mortality index. Suppose that the K-call is issued at time $t_{0}$, matures at time $T$, and has a strike value of $K$. The payoff of this K-call at maturity is

$$
\mathcal{C}^{(i)}(T, K)=\max \left(\kappa_{T}^{(i)}-K, 0\right)
$$

per $\$ 1$ notional, and the price of this K-call at time $t$ for $t_{0} \leq t<T$ is

$$
\begin{aligned}
C_{t}^{(i)}(T, K)= & \mathrm{E}_{t}^{(Q)}\left[\left(1+r_{f}\right)^{-(T-t)} \mathcal{C}^{(i)}(T, K)\right] \\
= & \left(1+r_{f}\right)^{-(T-t)}\left(\sqrt{\operatorname{Var}_{t}^{(Q)}\left[\kappa_{T}^{(i)}\right]} \phi\left(\frac{K-\mathrm{E}_{t}^{(Q)}\left[\kappa_{T}^{(i)}\right]}{\sqrt{\operatorname{Var}_{t}^{(Q)}\left[\kappa_{T}^{(i)}\right]}}\right)\right. \\
& -\left(K-\mathrm{E}_{t}^{(Q)}\left[\kappa_{T}^{(i)}\right]\right)\left(1-\Phi\left(\frac{K-\mathrm{E}_{t}^{(Q)}\left[\kappa_{T}^{(i)}\right]}{\sqrt{\operatorname{Var}_{t}^{(Q)}\left[\kappa_{T}^{(i)}\right]}}\right)\right)
\end{aligned}
$$


Finally, the time- $t$ values of the longevity delta and gamma for this K-call are

$$
\Delta_{t}^{(i, C)}(T, K)=\frac{\partial}{\partial \kappa_{t}^{(i)}} C_{t}^{(i)}(T, K)=\left(1+r_{f}\right)^{-(T-t)}\left(1-\Phi\left(\frac{K-\mathrm{E}_{t}^{(Q)}\left[\kappa_{T}^{(i)}\right]}{\sqrt{\operatorname{Var}_{t}^{(Q)}\left[\kappa_{T}^{(i)}\right]}}\right)\right)
$$

and

$$
\Gamma_{t}^{(i, C)}(T, K)=\frac{\partial}{\partial \kappa_{t}^{(i)}} \Delta_{t}^{(i, C)}(T, K)=\left(1+r_{f}\right)^{-(T-t)} \frac{1}{\sqrt{\operatorname{Var}_{t}^{(Q)}\left[\kappa_{T}^{(i)}\right]}} \phi\left(\frac{K-\mathrm{E}_{t}^{(Q)}\left[\kappa_{T}^{(i)}\right]}{\sqrt{\operatorname{Var}_{t}^{(Q)}\left[\kappa_{T}^{(i)}\right]}}\right)
$$

respectively. In the above, $\mathrm{E}_{t}^{(Q)}\left[\kappa_{T}^{(i)}\right]$ and $\operatorname{Var}_{t}^{(Q)}\left[\kappa_{T}^{(i)}\right]$ are given by equations (5) and (6), respectively, according to the model assumptions made. The full derivation of $C_{t}^{(i)}(T, K), \Delta_{t}^{(i, C)}(T, K)$ and $\Gamma_{t}^{(i, C)}(T, K)$ is presented in Appendix A.

Recall that pension plan sponsors are typically subject to downside K1 and K2 risks. As such, they may mitigate their risk exposures by taking a short position in K1- and K2-calls. Likewise, life insurers, who are typically subject to an upside $\mathrm{K} 1$ risk and a downside $\mathrm{K} 2$ risk, may reduce their risk exposures by taking a long position in a K1-call and a short position in a K2-call.

\subsection{K-put}

Consider a (European) K-put on the $i$ th CBD mortality index. Suppose again that the K-put is issued at time $t_{0}$, matures at time $T$, and has a strike value of $K$. The payoff of this K-put at maturity is

$$
\mathcal{P}^{(i)}(T, K)=\max \left(K-\kappa_{T}^{(i)}, 0\right)
$$

per $\$ 1$ notional, and the price of this K-put at time $t$ for $t_{0} \leq t<T$ is

$$
\begin{aligned}
P_{t}^{(i)}(T, K)= & \mathrm{E}_{t}^{(Q)}\left[\left(1+r_{f}\right)^{-(T-t)} \mathcal{P}^{(i)}(T, K)\right] \\
= & \left(1+r_{f}\right)^{-(T-t)}\left(\sqrt{\operatorname{Var}_{t}^{(Q)}\left[\kappa_{T}^{(i)}\right]} \phi\left(\frac{K-\mathrm{E}_{t}^{(Q)}\left[\kappa_{T}^{(i)}\right]}{\sqrt{\operatorname{Var}_{t}^{(Q)}\left[\kappa_{T}^{(i)}\right]}}\right)\right. \\
& \left.+\left(K-\mathrm{E}_{t}^{(Q)}\left[\kappa_{T}^{(i)}\right]\right) \Phi\left(\frac{K-\mathrm{E}_{t}^{(Q)}\left[\kappa_{T}^{(i)}\right]}{\sqrt{\operatorname{Var}_{t}^{(Q)}\left[\kappa_{T}^{(i)}\right]}}\right)\right) .
\end{aligned}
$$

Finally, the time- $t$ values of the longevity delta and gamma of this K-put are given by

$$
\Delta_{t}^{(i, P)}(T, K)=\frac{\partial}{\partial \kappa_{t}^{(i)}} P_{t}^{(i)}(T, K)=-\left(1+r_{f}\right)^{-(T-t)} \Phi\left(\frac{K-\mathrm{E}_{t}^{(Q)}\left[\kappa_{T}^{(i)}\right]}{\sqrt{\operatorname{Var}_{t}^{(Q)}\left[\kappa_{T}^{(i)}\right]}}\right)
$$

and

$$
\Gamma_{t}^{(i, P)}(T, K)=\frac{\partial}{\partial \kappa_{t}^{(i)}} \Delta_{t}^{(i, P)}(T, K)=\left(1+r_{f}\right)^{-(T-t)} \frac{1}{\sqrt{\operatorname{Var}_{t}^{(Q)}\left[\kappa_{T}^{(i)}\right]}} \phi\left(\frac{K-\mathrm{E}_{t}^{(Q)}\left[\kappa_{T}^{(i)}\right]}{\sqrt{\operatorname{Var}_{t}^{(Q)}\left[\kappa_{T}^{(i)}\right]}}\right)
$$


respectively. In the above, $\mathrm{E}_{t}^{(Q)}\left[\kappa_{T}^{(i)}\right]$ and $\operatorname{Var}_{t}^{(Q)}\left[\kappa_{T}^{(i)}\right]$ are given by equations (5) and (6), respectively, according to the model assumptions made. The full derivation of $P_{t}^{(i)}(T, K), \Delta_{t}^{(i, P)}(T, K)$ and $\Gamma_{t}^{(i, P)}(T, K)$ is presented in Appendix B.

Pension plan sponsors, who are typically subject to downside K1 and K2 risks, may mitigate their risk exposures by taking a long position in K1- and K2-puts. Life insurers, who are typically subject to an upside $\mathrm{K} 1$ risk and a downside K2 risk, may reduce their risk exposures by taking a short position in a K1-put and a long position in a K2-put.

\subsection{The Put-Call Parity}

The put-call parity says that a portfolio of a long call option and a short put option is equivalent to (and hence has the same value as) a forward contract at this strike price (fixed leg) and expiry. This important relationship also holds for options and forwards written on the CBD mortality indexes.

In terms of payoffs, for fixed $i, K$ and $T$, we have

$$
\begin{aligned}
\mathcal{C}^{(i)}(T, K)-\mathcal{P}^{(i)}(T, K) & =\max \left(\kappa_{T}^{(i)}-K, 0\right)-\max \left(K-\kappa_{T}^{(i)}, 0\right) \\
& =\kappa_{T}^{(i)}-K \\
& =-\mathcal{F}^{(i)}(T, K),
\end{aligned}
$$

which corresponds to the per unit notional payoff of the forward contract with parameters $i, K$ and $T$ from the fixed rate payer's perspective.

In terms of values, for fixed $i, K$ and $T$, we have

$$
\begin{aligned}
& C_{t}^{(i)}(T, K)-P_{t}^{(i)}(T, K) \\
= & \left(1+r_{f}\right)^{-(T-t)}\left(\sqrt{\operatorname{Var}_{t}^{(Q)}\left[\kappa_{T}^{(i)}\right]} \phi\left(\frac{K-\mathrm{E}_{t}^{(Q)}\left[\kappa_{T}^{(i)}\right]}{\sqrt{\operatorname{Var}_{t}^{(Q)}\left[\kappa_{T}^{(i)}\right]}}\right)-\left(K-\mathrm{E}_{t}^{(Q)}\left[\kappa_{T}^{(i)}\right]\right)\left(1-\Phi\left(\frac{K-\mathrm{E}_{t}^{(Q)}\left[\kappa_{T}^{(i)}\right]}{\sqrt{\operatorname{Var}_{t}^{(Q)}\left[\kappa_{T}^{(i)}\right]}}\right)\right)\right. \\
& -\left(1+r_{f}\right)^{-(T-t)}\left(\sqrt{\operatorname{Var}_{t}^{(Q)}\left[\kappa_{T}^{(i)}\right]} \phi\left(\frac{K-\mathrm{E}_{t}^{(Q)}\left[\kappa_{T}^{(i)}\right]}{\sqrt{\operatorname{Var}_{t}^{(Q)}\left[\kappa_{T}^{(i)}\right]}}\right)+\left(K-\mathrm{E}_{t}^{(Q)}\left[\kappa_{T}^{(i)}\right]\right) \Phi\left(\frac{K-\mathrm{E}_{t}^{(Q)}\left[\kappa_{T}^{(i)}\right]}{\left.\sqrt{\operatorname{Var}_{t}^{(Q)}\left[\kappa_{T}^{(i)}\right.}\right]}\right)\right. \\
= & -(1+r)^{-(T-t)}\left(K-\mathrm{E}_{t}^{(Q)}\left[\kappa_{T}^{(i)}\right]\right) \\
= & -F_{t}^{(i)}(T, K)
\end{aligned}
$$

for $t_{0} \leq t<T$. Note that $-F_{t}^{(i)}(T, K)$ corresponds to the per unit notional time- $t$ value of the forward contract with parameters $i, K$ and $T$ from the fixed rate payer's perspective.

\subsection{Moneyness}

Consider K-options with a strike value of $K$ and maturity date of $T$. At any time $t$, a K-option is called at-the-money if $K=\mathrm{E}_{t}^{(Q)}\left[\kappa_{T}^{(i)}\right]$. If $K<\mathrm{E}_{t}^{(Q)}\left[\kappa_{T}^{(i)}\right]$, then the K-call is called in-the-money 
while the K-put is called out-of-the-money. Similarly, if $K>\mathrm{E}_{t}^{(Q)}\left[\kappa_{T}^{(i)}\right]$, then the K-put is called in-the-money while the K-call is called out-of-the-money. It is obvious that the moneyness of a K-option depends on the following two factors:

1. the strike value $K$, and

2. $\mathrm{E}_{t}^{(Q)}\left[\kappa_{T}^{(i)}\right]$, which further depends on $\kappa_{t}^{(i)}$.

To facilitate exposition, we define the following moneyness metric:

$$
\text { Moneyness }=\frac{K-\mathrm{E}_{t}^{(Q)}\left[\kappa_{T}^{(i)}\right]}{\sqrt{\operatorname{Var}_{t}^{(Q)}\left[\kappa_{T}^{(i)}\right]}}
$$

which measures the difference between $K$ and $\mathrm{E}_{t}^{(Q)}\left[\kappa_{T}^{(i)}\right]$ relative to the standard deviation of $\kappa_{T}^{(i)}$ given the information up to and including time $t$. Although the notion of moneyness does not apply to forward contracts, for ease of exposition, in our numerical illustrations we may express the fixed leg $K$ of a K-forward in terms of 'Moneyness' through equation (8).

When 'Moneyness' equals zero (i.e., $K=\mathrm{E}_{t}^{(Q)}\left[\kappa_{T}^{(i)}\right]$ ), the time- $t$ prices of the K-call and K-put take the same value:

$$
C_{t}^{(i)}(T, K)=P_{t}^{(i)}(T, K)=\left(1+r_{f}\right)^{-(T-t)} \sqrt{\frac{\operatorname{Var}_{t}^{(Q)}\left[\kappa_{T}^{(i)}\right]}{2 \pi}} .
$$

This interesting property can be explained as follows. When $K=\mathrm{E}_{t}^{(Q)}\left[\kappa_{T}^{(i)}\right]$, according to equations (4) and (5), the payoffs of the K-call and the K-put are

$$
\max \left(\kappa_{T}^{(i)}-K, 0\right)=\max \left(\sum_{k=1}^{T-t} \tilde{\epsilon}_{t+k}^{(i)}, 0\right), \quad \text { and } \quad \max \left(K-\kappa_{T}^{(i)}, 0\right)=\max \left(-\sum_{k=1}^{T-t} \tilde{\epsilon}_{t+k}^{(i)}, 0\right)
$$

respectively. Since the distribution of $\sum_{k=1}^{T-t} \tilde{\epsilon}_{t+k}^{(i)}$ is symmetric, the payoffs from both options are identically distributed, which in turn means that the options must have the same price.

If the K-call is deeply in-the-money at time $t$ (i.e., Moneyness $\rightarrow-\infty$ ), then its price is close to $-F_{t}^{(i)}(T, K)$ (i.e., the time- $t$ value of an otherwise identical K-forward from the fixed rate payer's perspective). This outcome is because when 'Moneyness' becomes very negative, we have $\phi\left(\frac{K-\mathrm{E}_{t}^{(Q)}\left[\kappa_{T}^{(i)}\right]}{\sqrt{\operatorname{Var}_{t}^{(Q)}\left[\kappa_{T}^{(i)}\right]}}\right) \approx 0$ and $\left.\Phi\left(\frac{K-\mathrm{E}_{t}^{(Q)}\left[\kappa_{T}^{(i)}\right]}{\sqrt{\operatorname{Var}_{t}^{(Q)}\left[\kappa_{T}^{(i)}\right]}}\right)\right) \approx 0$, so that, according to equation (7), the price of the K-call becomes

$$
C_{t}^{(i)}(T, K) \approx-\left(1+r_{f}\right)^{-(T-t)}\left(K-\mathrm{E}_{t}^{(Q)}\left[\kappa_{T}^{(i)}\right]\right)=-F_{t}^{(i)}(T, K)
$$

On the other hand, if the $\mathrm{K}$-call is deeply out-of-the-money at time $t$ (i.e., Moneyness $\rightarrow+\infty$ ), then its price is close to 0 . This outcome is because when 'Moneyness' becomes very large, we 
have $\phi\left(\frac{K-\mathrm{E}_{t}^{(Q)}\left[\kappa_{T}^{(i)}\right]}{\sqrt{\operatorname{Var}_{t}^{(Q)}\left[\kappa_{T}^{(i)}\right]}}\right) \approx 0$ and $\left.\Phi\left(\frac{K-\mathrm{E}_{t}^{(Q)}\left[\kappa_{T}^{(i)}\right]}{\sqrt{\operatorname{Var}_{t}^{(Q)}\left[\kappa_{T}^{(i)}\right]}}\right)\right) \approx 1$, so that, according to equation (7), the price of the K-call becomes

$$
C_{t}^{(i)}(T, K) \approx 0
$$

Using similar arguments, the price of the K-put becomes

$$
P_{t}^{(i)}(T, K) \approx\left(1+r_{f}\right)^{-(T-t)}\left(K-\mathrm{E}_{t}^{(Q)}\left[\kappa_{T}^{(i)}\right]\right)=F_{t}^{(i)}(T, K)
$$

when it is deeply in-the-money (i.e., Moneyness $\rightarrow+\infty$ ), and becomes

$$
P_{t}^{(i)}(T, K) \approx 0
$$

when it is deeply out-of-the-money (i.e., Moneyness $\rightarrow-\infty$ ).

The left panels of Figure 3 depict the relationship between moneyness and price, on the basis of the assumed market prices of risk and parameters that are estimated to real data. ${ }^{5}$ All of the aforementioned properties can be observed from the diagram.

In the middle and right panels of Figure 3, we show the relationships between moneyness and the longevity Greeks. The following properties (which can be deduced easily from the formulas presented in Sections 4.1, 4.2 and 4.3) can be observed:

1. The longevity delta of a K-forward is insensitive to its strike value $K$, and the longevity gamma of a K-forward is always 0 .

2. At any given time point $t$, the difference between the longevity deltas of a K-call and Kput with the same strike value and time-to-maturity $(T-t)$ is a constant that equals to $\left(1+r_{f}\right)^{-(T-t)}$.

3. At any given time point, the longevity gammas of a K-call and K-put with the same strike value and time-to-maturity are identical.

\section{$5 \quad$ The Liability Being Hedged}

\subsection{Specification}

It is assumed that the liability being hedged is a whole life annuity-immediate of $\$ 1$ that is sold to individuals aged $x_{0}$ at time $t_{0}$. Discounted to time $t$ at an effective interest rate of $r$ per annum, the sum of the annuity payments beyond time $t$ (per surviving annuitant at time $t$ ) is

$$
\mathcal{L}(t, r)=\sum_{s=1}^{\omega-x_{0}-t+t_{0}}(1+r)^{-s} \mathcal{S}_{x_{0}+t-t_{0}, t}(s), \quad t=t_{0}, t_{0}+1, \ldots, t_{0}+\omega-x_{0}-1,
$$

\footnotetext{
${ }^{5}$ Parameter estimation is discussed in Section 7.1.
} 

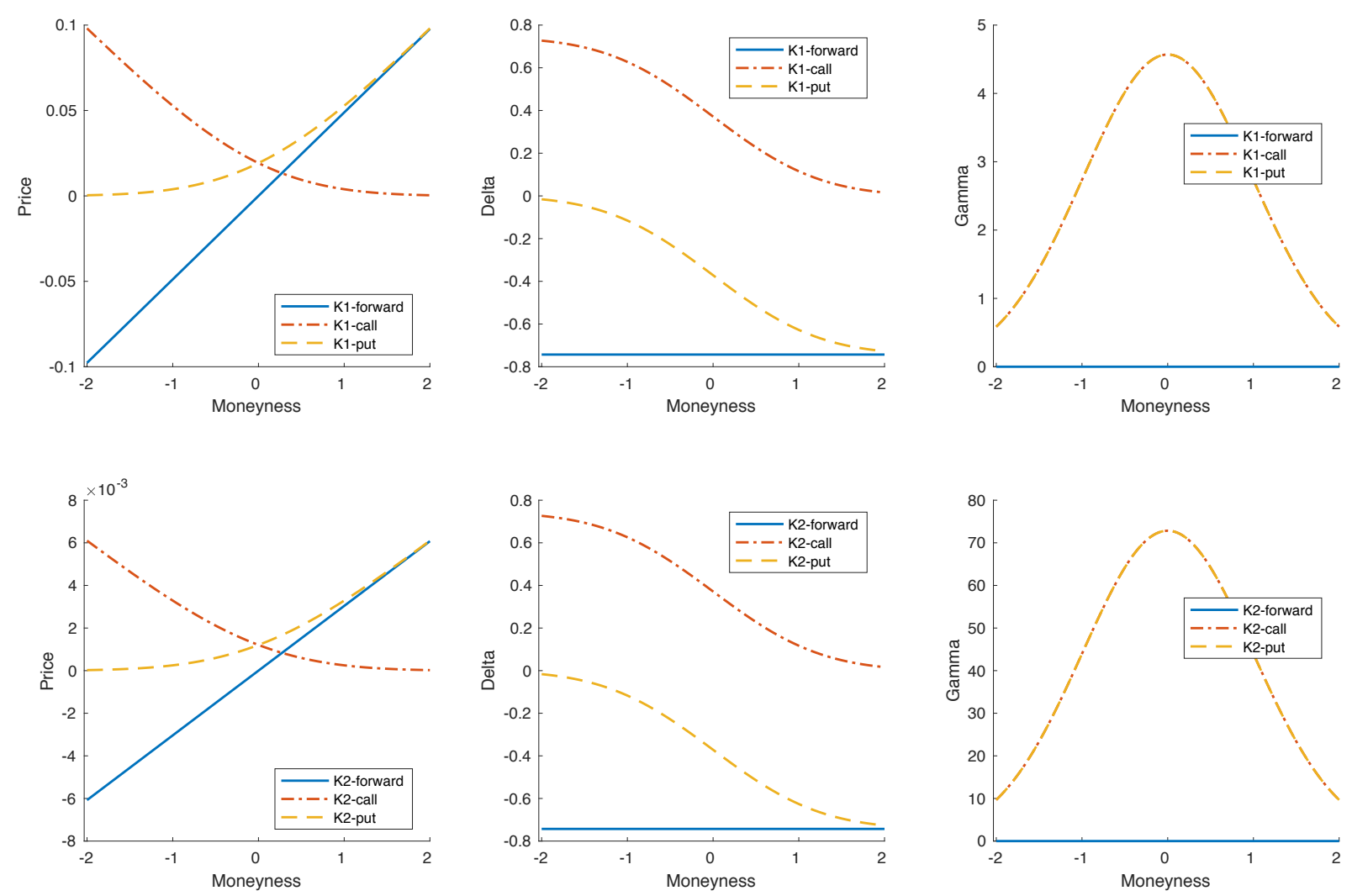

Figure 3: Time-t prices, deltas and gammas of K-calls, K-puts and K-forwards with different degrees of moneyness. The values shown are calculated with $r_{f}=0.02, T-t=15, \mathrm{E}_{t}^{(Q)}\left[\kappa_{T}^{(1)}\right]=$ $-5.6304, \sqrt{\operatorname{Var}_{t}^{(Q)}\left[\kappa_{T}^{(1)}\right]}=6.4874 \times 10^{-2}, \mathrm{E}_{t}^{(Q)}\left[\kappa_{T}^{(2)}\right]=0.1087, \sqrt{\operatorname{Var}_{t}^{(Q)}\left[\kappa_{T}^{(2)}\right]}=4.0693 \times 10^{-3}$, and $K=\mathrm{E}_{t}^{(Q)}\left[\kappa_{T}^{(i)}\right]+$ Moneyness $\times \sqrt{\operatorname{Var}_{t}^{(Q)}\left[\kappa_{T}^{(i)}\right]}$.

where $\omega$ is the limiting age, and

$$
\mathcal{S}_{x, t}(s)=\prod_{u=1}^{s}\left(1+\exp \left(\boldsymbol{\beta}_{x+u-1} \boldsymbol{\kappa}_{t+u}\right)\right)^{-1}
$$

with $\boldsymbol{\beta}_{x}=(1, x-\bar{x})$ represents the ex post probability that an individual aged $x$ at time $t$ would have survived to time $t+s .^{6}$

The time- $t$ value of the (unpaid) annuity liability is the risk-neutral expectation of $\mathcal{L}\left(t, r_{f}\right)$, given information up to and including time $t$; that is,

$$
L_{t}=\mathrm{E}_{t}^{(Q)}\left[\mathcal{L}\left(t, r_{f}\right)\right]=\sum_{s=1}^{\omega-x_{0}-t+t_{0}}\left(1+r_{f}\right)^{-s} \mathrm{E}_{t}^{(Q)}\left[\mathcal{S}_{x_{0}+t-t_{0}, t}(s)\right],
$$

\footnotetext{
${ }^{6}$ We do not incorporate sampling risk (which arises from the fact that the portfolio of annuity has a finite size) into the definition of $\mathcal{S}_{x, t}(s)$ and subsequent analyses. This piece of uncertainty is diversifiable and is not intended to be hedged using the index-based hedging instruments.
} 
for $t=t_{0}, t_{0}+1, \ldots, t_{0}+\omega-x_{0}-1$. It is obvious that $L_{t}$ depends on the values of $\mathrm{E}_{t}^{(Q)}\left[\mathcal{S}_{x, t}(s)\right]$ for $s=1, \ldots, \omega-x_{0}-t+t_{0}$ and $x=x_{0}+t-t_{0}$. Furthermore, given the Markov property of the bivariate random walk (under the risk-neutral probability measure), the value of $\mathrm{E}_{t}^{(Q)}\left[\mathcal{S}_{x, t}(s)\right]$ depends exclusively on the values of $\kappa_{t}^{(1)}$ and $\kappa_{t}^{(2)}$.

In the following situations, nested simulations are required to estimate the exact values of $L_{t}$ for $t>t_{0}$ :

1. Dynamic hedging

For each of the $N$ simulated mortality scenarios (under the real-world probability measure) for evaluating hedge effectiveness, $M$ simulations (under the risk-neutral probability measure) are needed to calculate $L_{t}$ for each time point $t$ at which the hedge is adjusted. The entire procedure involves $N \times M \times k$ simulations, where $k$ is the number of time points at which the hedge is adjusted.

2. Evaluation of the $\tau$-year ahead Value-at-Risk

To obtain an empirical distribution of $L_{\tau}$ from which the $\tau$-year ahead Value-at-Risk can be estimated, we need $M$ simulations (under the risk-neutral probability measure) to calculate $L_{\tau}$ for each of the $N$ simulated mortality scenarios (under the real-world probability measure) for evaluating hedge effectiveness. The entire procedure entails $N \times M$ simulations.

In the next sub-section, we present a method to approximate the values of $\mathrm{E}_{t}^{(Q)}\left[\mathcal{S}_{x, t}(s)\right]$ for $s=1, \ldots, \omega-x_{0}-t+t_{0}$ and $x=x_{0}+t-t_{0}$, so that the computationally demanding nested simulations can be avoided.

\subsection{The Approximation Method}

Following Cairns (2011), we apply a first-order Taylor's series approximation to the probit transform of $\mathrm{E}_{t}^{(Q)}\left[\mathcal{S}_{x, t}(s)\right]$; that is,

$$
\Phi^{-1}\left(\mathrm{E}_{t}^{(Q)}\left[\mathcal{S}_{x, t}(s)\right]\right) \approx d_{x, t, 0}(s)+d_{x, t, 1}^{(1)}(s)\left(\kappa_{t}^{(1)}-\hat{\kappa}_{t}^{(1)}\right)+d_{x, t, 1}^{(2)}\left(\kappa_{t}^{(2)}-\hat{\kappa}_{t}^{(2)}\right),
$$

where $\Phi$ is the standard normal distribution function, $d_{x, t, 0}(s)=\Phi^{-1}\left(\mathrm{E}_{t}^{(Q)}\left[\mathcal{S}_{x, t}(s)\right]\right)$,

$$
d_{x, t, 1}^{(1)}(s)=\frac{\partial}{\partial \kappa_{t}^{(1)}} \Phi^{-1}\left(\mathrm{E}_{t}^{(Q)}\left[\mathcal{S}_{x, t}(s)\right]\right)
$$

and

$$
d_{x, t, 1}^{(2)}(s)=\frac{\partial}{\partial \kappa_{t}^{(2)}} \Phi^{-1}\left(\mathrm{E}_{t}^{(Q)}\left[\mathcal{S}_{x, t}(s)\right]\right) .
$$

The values of $d_{x, t, 0}(s), d_{x, t, 1}^{(1)}(s)$ and $d_{x, t, 1}^{(2)}(s)$ are evaluated at $\kappa_{t}^{(1)}=\hat{\kappa}_{t}^{(1)}$ and $\kappa_{t}^{(2)}=\hat{\kappa}_{t}^{(2)}$. We set $\hat{\kappa}_{t}^{(1)}$ and $\hat{\kappa}_{t}^{(2)}$ to $\mathrm{E}_{t_{0}}^{(P)}\left[\kappa_{t}^{(1)}\right]$ and $\mathrm{E}_{t_{0}}^{(P)}\left[\kappa_{t}^{(2)}\right]$, respectively, where $\mathrm{E}_{t}^{(P)}[\cdot]$ represents the expectation under the real-world probability measure, given information up to and including time $t$. 
To derive $d_{x, t, 1}^{(1)}(s)$ and $d_{x, t, 1}^{(2)}(s)$, we use the fact that

$$
\frac{\partial \Phi^{-1}(f(x))}{\partial x}=\frac{1}{\phi\left(\Phi^{-1}(f(x))\right)} \frac{\partial f(x)}{\partial x}
$$

where $\phi$ is the standard normal probability density function. It follows that

$$
\begin{aligned}
d_{x, t, 1}^{(i)}(s) & =\frac{1}{\phi\left(\Phi^{-1}\left(\mathrm{E}_{t}^{(Q)}\left[\mathcal{S}_{x, t}(s)\right]\right)\right)}\left(\frac{\partial}{\partial \kappa_{t}^{(i)}} \mathrm{E}_{t}^{(Q)}\left[\mathcal{S}_{x, t}(s)\right]\right) \\
& =\frac{1}{\phi\left(\Phi^{-1}\left(\mathrm{E}_{t}^{(Q)}\left[\mathcal{S}_{x, t}(s)\right]\right)\right)}\left(\frac{\partial}{\partial \kappa_{t}^{(i)}} \mathrm{E}_{t}^{(Q)}\left[\prod_{u=1}^{s}\left(1+\exp \left(\boldsymbol{\beta}_{x+u-1} \boldsymbol{\kappa}_{t+u}\right)\right)^{-1}\right]\right) \\
& =\frac{-1}{\phi\left(\Phi^{-1}\left(\mathrm{E}_{t}^{(Q)}\left[\mathcal{S}_{x, t}(s)\right]\right)\right)} \mathrm{E}_{t}^{(Q)}\left[\left(\mathcal{S}_{x, t}(s)\right)^{2} \frac{\partial}{\partial \kappa_{t}^{(i)}} \prod_{u=1}^{s}\left(1+\exp \left(\boldsymbol{\beta}_{x+u-1} \boldsymbol{\kappa}_{t+u}\right)\right)\right], \quad i=1,2 .
\end{aligned}
$$

In the above, $\frac{\partial}{\partial \kappa_{t}^{(i)}} \prod_{u=1}^{s}\left(1+\exp \left(\boldsymbol{\beta}_{x+u-1} \boldsymbol{\kappa}_{t+u}\right)\right)$ can be calculated recursively as

$$
\begin{aligned}
& \frac{\partial}{\partial \kappa_{t}^{(i)}} \prod_{u=1}^{s}\left(1+\exp \left(\boldsymbol{\beta}_{x+u-1} \boldsymbol{\kappa}_{t+u}\right)\right) \\
= & \begin{cases}\frac{\partial \boldsymbol{\beta}_{x} \boldsymbol{\kappa}_{t+1}}{\partial \kappa_{t}^{(i)}} \exp \left(\boldsymbol{\beta}_{x} \boldsymbol{\kappa}_{t+1}\right), & s=1 \\
\frac{\partial \boldsymbol{\beta}_{x+u-1} \boldsymbol{\kappa}_{t+u}}{\partial \kappa_{t}^{(i)}} \exp \left(\boldsymbol{\beta}_{x+s-1} \boldsymbol{\kappa}_{t+s}\right) \prod_{u=1}^{s-1}\left(1+\exp \left(\boldsymbol{\beta}_{x+u-1} \boldsymbol{\kappa}_{t+u}\right)\right) & , \\
+\left(1+\exp \left(\boldsymbol{\beta}_{x+s-1} \boldsymbol{\kappa}_{t+s}\right)\right) \frac{\partial}{\partial \kappa_{t}^{(i)}} \prod_{u=1}^{s-1}\left(1+\exp \left(\boldsymbol{\beta}_{x+u-1} \boldsymbol{\kappa}_{t+u}\right)\right), & s>1\end{cases}
\end{aligned}
$$

where, according to equation (4),

$$
\frac{\partial \boldsymbol{\beta}_{x+s-1} \boldsymbol{\kappa}_{t+s}}{\partial \kappa_{t}^{(1)}}=\frac{\partial \kappa_{t+s}^{(1)}}{\partial \kappa_{t}^{(1)}}+(x+s-1-\bar{x}) \frac{\partial \kappa_{t+s}^{(2)}}{\partial \kappa_{t}^{(1)}}=1
$$

and

$$
\frac{\partial \boldsymbol{\beta}_{x+s-1} \boldsymbol{\kappa}_{t+s}}{\partial \kappa_{t}^{(2)}}=\frac{\partial \kappa_{t+s}^{(1)}}{\partial \kappa_{t}^{(2)}}+(x+s-1-\bar{x}) \frac{\partial \kappa_{t+s}^{(2)}}{\partial \kappa_{t}^{(2)}}=x+s-1-\bar{x}
$$

for $s \geq 1$.

\subsection{Deriving Longevity Deltas}

The longevity deltas of the (unpaid) annuity liability are defined as the first partial derivatives of $L_{t}$ with respect to $\kappa_{t}^{(1)}$ and $\kappa_{t}^{(2)}$; that is,

$$
\Delta_{t}^{(i, L)}=\frac{\partial L_{t}}{\partial \kappa_{t}^{(i)}}=\sum_{s=1}^{\omega-x_{0}-t+t_{0}}\left(1+r_{f}\right)^{-s} \frac{\partial}{\partial \kappa_{t}^{(i)}} \mathrm{E}_{t}^{(Q)}\left[\mathcal{S}_{x_{0}+t-t_{0}, t}(s)\right], \quad i=1,2 .
$$


To determine $\Delta_{t}^{(i, L)}$, we need to compute the values of $\frac{\partial}{\partial \kappa_{t}^{(i)}} \mathrm{E}_{t}^{(Q)}\left[\mathcal{S}_{x, t}(s)\right]$ for $s=1, \ldots, \omega-$ $x_{0}-t+t_{0}$ and $x=x_{0}+t-t_{0}$. When $t=t_{0}$, we have

$$
\frac{\partial}{\partial \kappa_{t}^{(i)}} \mathrm{E}_{t}^{(Q)}\left[\mathcal{S}_{x, t}(s)\right]=\mathrm{E}_{t}^{(Q)}\left[\left(\mathcal{S}_{x, t}(s)\right)^{2} \frac{\partial}{\partial \kappa_{t}^{(i)}} \prod_{u=1}^{s}\left(1+\exp \left(\boldsymbol{\beta}_{x+u-1} \boldsymbol{\kappa}_{t+u}\right)\right)\right], \quad i=1,2,
$$

where $\frac{\partial}{\partial \kappa_{t}^{(i)}} \prod_{u=1}^{s}\left(1+\exp \left(\boldsymbol{\beta}_{x+u-1} \boldsymbol{\kappa}_{t+u}\right)\right)$ can be obtained using equation (9). When $t>t_{0}$, we use the approximation formula to get

$$
\begin{aligned}
\frac{\partial}{\partial \kappa_{t}^{(i)}} \mathrm{E}_{t}^{(Q)}\left[\mathcal{S}_{x, t}(s)\right] & \approx \frac{\partial}{\partial \kappa_{t}^{(i)}} \Phi\left(d_{x, t, 0}(s)+d_{x, t, 1}^{(1)}(s)\left(\kappa_{t}^{(1)}-\hat{\kappa}_{t}^{(1)}\right)+d_{x, t, 1}^{(2)}(s)\left(\kappa_{t}^{(2)}-\hat{\kappa}_{t}^{(2)}\right)\right) \\
& =\phi\left(d_{x, t, 0}(s)+d_{x, t, 1}^{(1)}(s)\left(\kappa_{t}^{(1)}-\hat{\kappa}_{t}^{(1)}\right)+d_{x, t, 1}^{(2)}(s)\left(\kappa_{t}^{(2)}-\hat{\kappa}_{t}^{(2)}\right)\right) d_{x, t, 1}^{(i)}(s)
\end{aligned}
$$

for $i=1,2$.

\subsection{Accuracy of the Approximation}

We now evaluate the accuracy of the approximation method discussed in Section 5.2. The evaluation is based a (real-world) Cairns-Blake-Dowd model that is fitted to historical data up to and including year $t_{0}=2013$ and its risk-neutral counterpart that is defined using $\lambda^{(1)}=\lambda^{(2)}=0.175$ (see Section 7.1 for further details).

Figure 4 demonstrates the accuracy of the approximation for $L_{t_{0}+1}$, a quantity that is highly relevant to the calculation of the 1-year Value-at-Risk and the evaluation of an annually adjusted dynamic hedge. ${ }^{7}$ In both panels, the dots represent 1,000 simulated pairs of $\left(\kappa_{t_{0}+1}^{(1)}, \kappa_{t_{0}+1}^{(2)}\right)$, so the cloud of dots can be understood as the possible range of $\left(\kappa_{t_{0}+1}^{(1)}, \kappa_{t_{0}+1}^{(2)}\right){ }^{8}$ In the left panel, the solid blue lines represent the 'true' values which are calculated by full (nested) simulations, whereas the dashed red lines represent the approximated values. It can be observed that the gaps between the solid and dashed lines are very small, indicating that the approximation is very accurate. In the right panel, the contour lines represent the absolute percentage errors in approximating $L_{t_{0}+1}$. Within the boundary of the cloud of dots, the absolute percentage errors are no greater than $0.03 \%$, further confirming that the approximation is highly accurate. The small absolute percentage errors also suggest that a higher-order approximation does not seem to be necessary.

In principle, we can apply similar approximations to the values of K-options, although such approximations are not necessary as exact analytical valuation formulas are available (see Section 4 ). For instance, when using a second-order approximation, the time- $t$ value of a K-put can be approximated as

$$
P_{t}^{(i)}(T, K) \approx c_{x, t, 0}(s)+c_{x, t, 1}^{(i)}(s)\left(\kappa_{t}^{(i)}-\hat{\kappa}_{t}^{(i)}\right)+c_{x, t, 2}^{(i)}(s)\left(\kappa_{t}^{(i)}-\hat{\kappa}_{t}^{(i)}\right)^{2},
$$

\footnotetext{
${ }^{7}$ The evaluation results for $L_{t_{0}+s}, s=2,3, \ldots$ are similar and therefore not shown.

${ }^{8}$ The 1,000 pairs of $\left(\kappa_{t_{0}+1}^{(1)}, \kappa_{t_{0}+1}^{(2)}\right)$ are simulated under the real-world probability measure.
} 

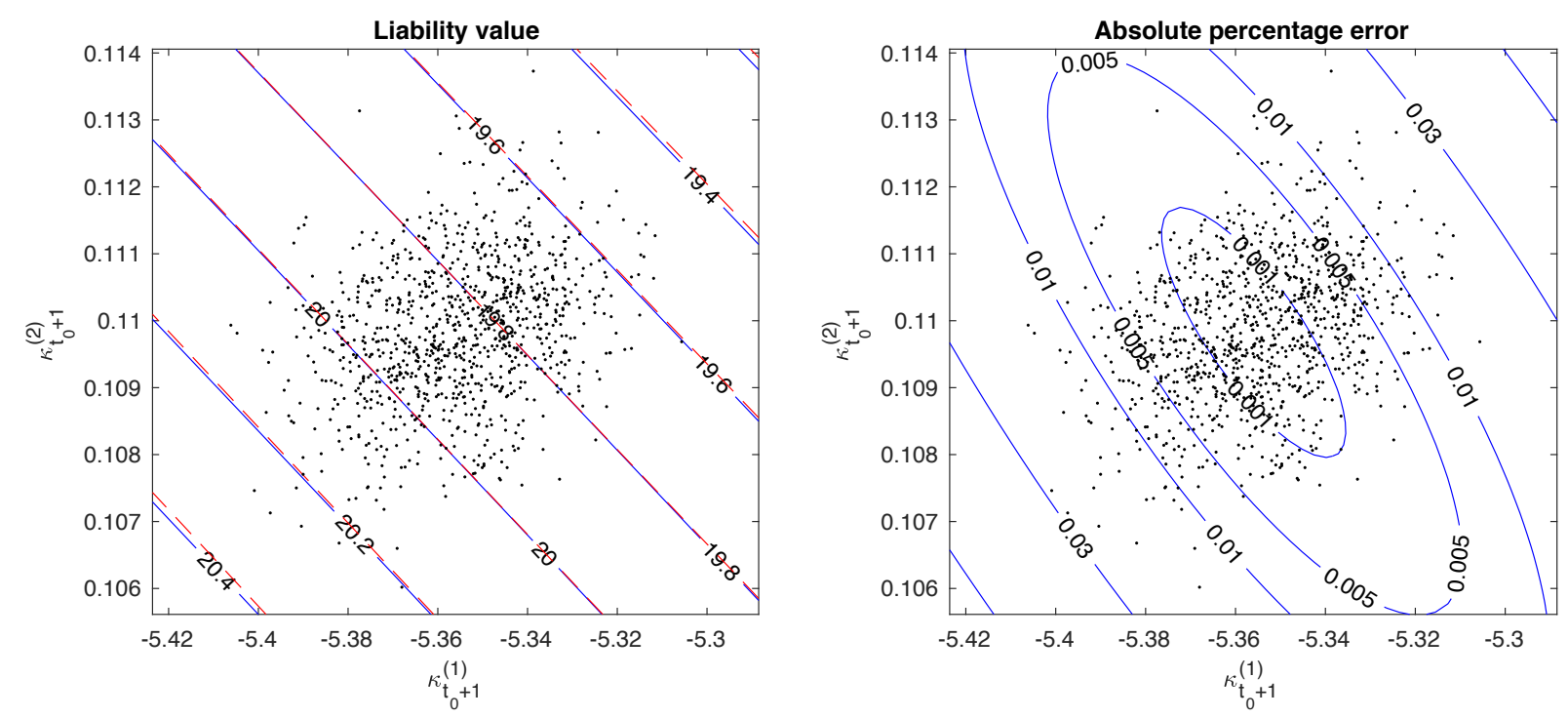

Figure 4: An illustration of the accuracy of the approximation for $L_{t_{0}+1}$. Left panel: contours represent the true (solid blue) and approximated (dashed red) values of $L_{t_{0}+1}$. Right panel: contours represent the absolute percentage error of the approximation.

where

$$
\begin{aligned}
c_{x, t, 0}(s) & =\mathrm{E}_{t}^{(Q)}\left[\left(1+r_{f}\right)^{-(T-t)} \max \left(K-\kappa_{T}^{(i)}, 0\right)\right], \\
c_{x, t, 1}^{(i)}(s) & =\frac{\partial}{\partial \kappa_{t}^{(i)}} \mathrm{E}_{t}^{(Q)}\left[\left(1+r_{f}\right)^{-(T-t)} \max \left(K-\kappa_{T}^{(i)}, 0\right)\right] \\
& =-\left(1+r_{f}\right)^{-(T-t)} \Phi\left(\frac{K-\mathrm{E}_{t}^{(Q)}\left[\kappa_{T}^{(i)}\right]}{\sqrt{\operatorname{Var}_{t}^{(Q)}\left[\kappa_{T}^{(i)}\right]}}\right) \\
& =\Delta_{t}^{(i, P)}(T, K)
\end{aligned}
$$

and

$$
\begin{aligned}
c_{x, t, 2}^{(i)}(s) & =\frac{\partial^{2}}{\partial\left(\kappa_{t}^{(i)}\right)^{2}} \mathrm{E}_{t}^{(Q)}\left[\left(1+r_{f}\right)^{-(T-t)} \max \left(K-\kappa_{T}^{(i)}, 0\right)\right] \\
& =\left(1+r_{f}\right)^{-(T-t)} \frac{1}{\sqrt{\operatorname{Var}_{t}^{(Q)}\left[\kappa_{T}^{(i)}\right]}} \phi\left(\frac{K-\mathrm{E}_{t}^{(Q)}\left[\kappa_{T}^{(i)}\right]}{\sqrt{\operatorname{Var}_{t}^{(Q)}\left[\kappa_{T}^{(i)}\right]}}\right) \\
& =\Gamma_{t}^{(i, P)}(T, K),
\end{aligned}
$$

with $c_{x, t, 0}(s), c_{x, t, 1}^{(i)}(s)$ and $c_{x, t, 2}^{(i)}(s)$ being calculated at $\kappa_{t}^{(1)}=\hat{\kappa}_{t}^{(1)}$ and $\kappa_{t}^{(2)}=\hat{\kappa}_{t}^{(2)}$.

Figure 5 demonstrates the accuracy of the approximation for $P_{t_{0}+1}^{(1)}\left(t_{0}+15, \mathrm{E}_{t_{0}}^{(Q)}\left[\kappa_{t_{0}+15}^{(1)}\right]\right)$, the time- $\left(t_{0}+1\right)$ value of a $\mathrm{K}$-put on the first CBD mortality index with a maturity date of $t_{0}+15$ 

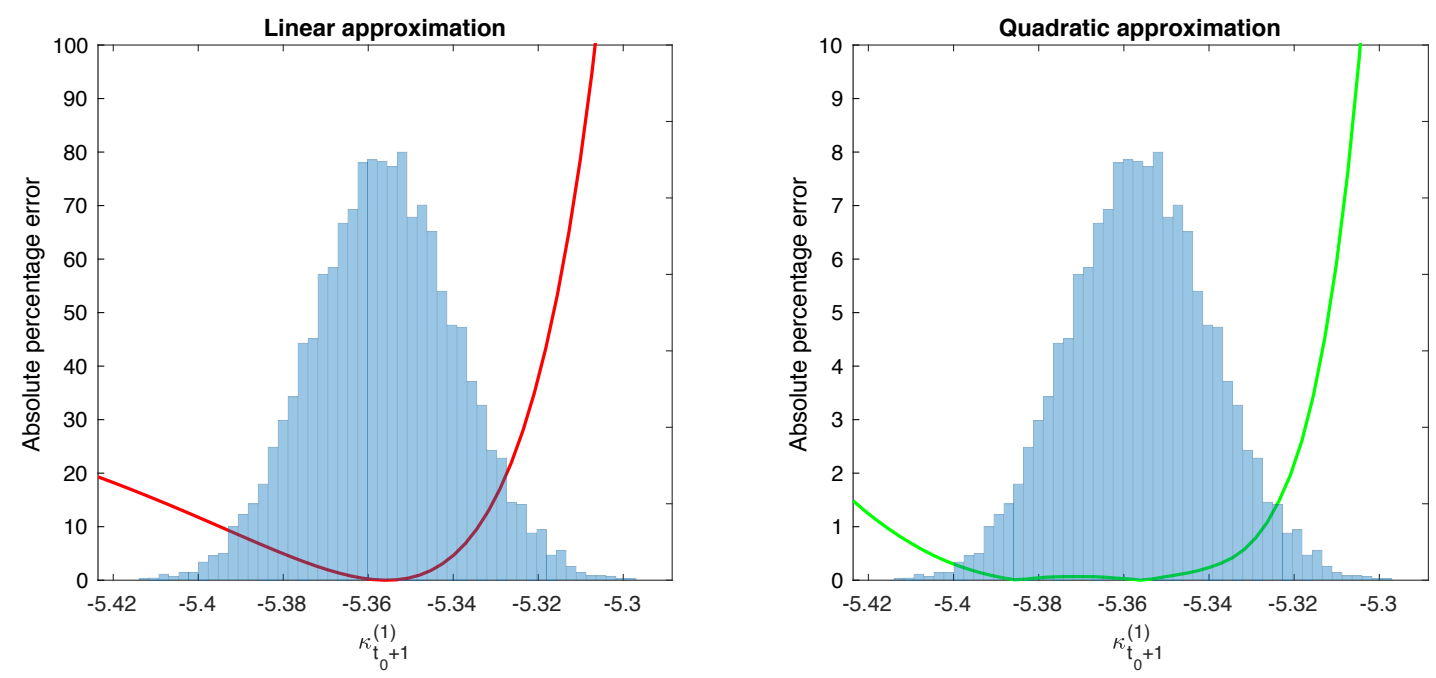

Figure 5: The absolute percentage errors resulting from the linear (left panel) and quadratic (right panel) approximations of the values of $P_{t_{0}+1}^{(1)}\left(t_{0}+15, \mathrm{E}_{t_{0}}^{(Q)}\left[\kappa_{t_{0}+15}^{(1)}\right]\right)$. The histogram represents the empirical distribution of $\kappa_{t_{0}+1}^{(1)}$ given the information up to and including time $t_{0}$ under the real-world probability measure.

and a strike value of $\mathrm{E}_{t_{0}}^{(Q)}\left[\kappa_{t_{0}+15}^{(1)}\right]$ (the option is at-the-money when it was issued at time $t_{0}$ ). In the figure, we show the absolute percentage errors (relative to the true values calculated using the analytical valuation formula) incurred in the linear approximation (left panel) and the quadratic approximation (right panel), along with the empirical distribution of $\kappa_{t_{0}+1}^{(1)}$ given information up to and including time $t_{0}$ under the real-world probability measure. By definition, the approximation is exact when $\kappa_{t_{0}+1}^{(1)}=\hat{\kappa}_{t_{0}+1}^{(1)}$. As $\kappa_{t_{0}+1}^{(1)}$ moves away from $\hat{\kappa}_{t_{0}+1}^{(1)}$, the absolute percentage error increases rapidly. For the linear approximation, the absolute percentage errors are higher than $100 \%$ for some simulated values of $\kappa_{t_{0}+1}^{(1)}$. The quadratic approximation is somewhat more accurate, but the absolute percentage error may still be higher than $10 \%$ in some circumstances. The results shown in Figure 5 confirm that Taylor's series approximations do not perform satisfactorily for the values of mortality derivatives with an option-like payoff, and highlight the importance of the exact analytical valuation formulas provided in Section 4.

\section{Developing Longevity Hedges}

\subsection{General Assumptions}

In this section, we develop delta longevity hedges using K-forwards and options. It is assumed that at any time, the hedge contains two instruments, one written on each of the two CBD mortality indexes.

All longevity hedges are assumed to be established at time $t_{0}$. Regardless of how the longevity 
hedge is formed, hedge effectiveness is measured in terms of Value-at-Risk, estimated using mortality scenarios that are simulated under the real-world probability measure (given information up to and including time $t_{0}$ ). Accordingly, all simulated cash flows should be discounted using the hedger's risk-adjusted interest rate $\left(r_{a}\right)$ instead of the risk-free interest rate $\left(r_{f}\right)$. Given the assumed value of $r_{f}$, the value of $r_{a}$ is obtained by solving the following equation numerically:

$$
\mathrm{E}_{t_{0}}^{(Q)}\left[\mathcal{L}\left(t_{0}, r_{f}\right)\right]=\mathrm{E}_{t_{0}}^{(P)}\left[\mathcal{L}\left(t_{0}, r_{a}\right)\right]
$$

To focus on our research goals, we put aside population basis risk (the risk that arises from the difference in mortality experience between the populations associated with the hedger's portfolio and the hedging instruments). Interested readers are referred to Li et al. (2017) for a recent study on population basis risk.

\subsection{Cash-flow Hedges}

This sub-section is devoted to cash-flow hedges, which focus on the variability of the (discounted) cash flows arising from the annuity liability and the hedging instruments.

\subsubsection{The Unhedged Position}

Without any longevity hedge in place, the sum of all annuity payments discounted to time $t_{0}$ at the risk-adjusted interest rate of $r_{a}$ is simply $\mathcal{L}\left(t_{0}, r_{a}\right)$.

We can straightforwardly simulate an empirical distribution of $\mathcal{L}\left(t_{0}, r_{a}\right)$ under the real-world probability measure. From the empirical distribution of $\mathcal{L}\left(t_{0}, r_{a}\right)$, we can obtain the Value-at-Risk (VaR) at the 99.5 confidence level (i.e., the 99.5 th percentile), which is then used to benchmark against the $\mathrm{VaR}$ values of the hedged positions.

\subsubsection{Static Hedges}

In a static hedge, no adjustment is made after time $t_{0}$ when the hedge is established. For the $i$ th instrument in the hedge, we use $T^{(i)}$ to represent its maturity date, $K^{(i)}$ to represent its strike value (if the instrument is a K-option) or its fixed leg (if the instrument is a K-forward).

The notional amounts of the two instruments are determined by matching the longevity deltas of the hedge portfolio and the liability being hedged. It follows that the notional amount of the $i$ th instrument is given by

$$
h^{(i)}=\frac{\Delta_{t_{0}}^{(i, L)}}{\Delta_{t_{0}}^{(i, H)}\left(T^{(i)}, K^{(i)}\right)}, \quad i=1,2
$$

where $H=F, C$, or $P$ depending on whether the $i$ th instrument is a K-forward, K-call or K-put. 
The hedging instruments incur a cash outflow (associated with their purchase prices) of

$$
\sum_{i=1}^{2} h^{(i)} H_{t_{0}}^{(i)}\left(T^{(i)}, K^{(i)}\right)
$$

at time $t_{0}$, and a cash inflow (associated with their payoffs) of

$$
\sum_{i=1}^{2} h^{(i)} \mathcal{H}^{(i)}\left(T^{(i)}, K^{(i)}\right)
$$

where $\mathcal{H}=\mathcal{F}, \mathcal{C}$, or $\mathcal{P}$ depending on whether the $i$ th instrument is a $\mathrm{K}$-forward, K-call or K-put. As a result, discounted to time $t_{0}$ at the risk-adjusted interest rate, the net cash outflows arising from the hedging instrument sum to

$$
\sum_{i=1}^{2} h^{(i)}\left(H_{t_{0}}^{(i)}\left(T^{(i)}, K^{(i)}\right)-\left(1+r_{a}\right)^{-\left(T^{(i)}-t_{0}\right)} \mathcal{H}^{(i)}\left(T^{(i)}, K^{(i)}\right)\right) .
$$

The performance of the hedge can be evaluated using the VaR at the $99.5 \%$ confidence level, obtained from the 99.5th percentile of the distribution of

$$
\mathcal{L}\left(t_{0}, r_{a}\right)+\sum_{i=1}^{2} h^{(i)}\left(H_{t_{0}}^{(i)}\left(T^{(i)}, K^{(i)}\right)-\left(1+r_{a}\right)^{-\left(T^{(i)}-t_{0}\right)} \mathcal{H}^{(i)}\left(T^{(i)}, K^{(i)}\right)\right),
$$

which represents the total discounted cash outflows of the hedged position. The empirical distribution of (12) can be obtained using simulated values of $\mathcal{L}\left(t_{0}, r_{a}\right)$ and $\mathcal{H}^{(i)}\left(T^{(i)}, K^{(i)}\right)$, given the information up to and including time $t_{0}$, under the real-world probability measure. The values of $h^{(i)}$ and $H_{t_{0}}^{(i)}\left(T^{(i)}, K^{(i)}\right)$ in expression (12) should be calculated using the exact formulas presented in Sections 4.1, 4.2, 4.3 and 5.3 prior to the simulation.

\subsubsection{Dynamic Hedges}

In a dynamic hedge, the hedge portfolio is adjusted annually until the annuity liability runs off completely. Specifically, it is assumed that at each time point $t$, for $t=t_{0}+1, \ldots, t_{0}+\omega-x_{0}-1$, the two hedging instruments purchased at the previous time point $(t-1)$ are closed out, and another two hedging instruments are purchased to suit the current longevity risk profile of the annuity liability.

For the $i$ th hedging instrument purchased at time $t$, where $t=t_{0}, \ldots, t_{0}+\omega-x_{0}-1$, we use

$T_{t}^{(i)}$ to denote its maturity date and $K_{t}^{(i)}$ to represent its strike value. The notional amounts of the hedging instruments purchased at time $t$ are determined by matching the time- $t$ values of the longevity deltas of the annuity liability and the hedging instruments. This strategy implies that the notional amount of the $i$ th instrument purchased at time $t$ is

$$
h_{t}^{(i)}=\frac{\Delta_{t}^{(i, L)}}{\Delta_{t}^{(i, H)}\left(T_{t}^{(i)}, K_{t}^{(i)}\right)}
$$


for $t=t_{0}, \ldots, t_{0}+\omega-x_{0}-1, i=1,2$, and $H=F, P$, or $C$.

The hedging instruments purchased at time $t$ incur a cash outflow (which is associated with their purchase prices) of

$$
\sum_{i=1}^{2} h_{t}^{(i)} H_{t}^{(i)}\left(T_{t}^{(i)}, K_{t}^{(i)}\right)
$$

at time $t$ and a cash inflow (which is associated with the prices for which they are sold) of

$$
\sum_{i=1}^{2} h_{t}^{(i)} H_{t+1}^{(i)}\left(T_{t}^{(i)}, K_{t}^{(i)}\right)
$$

when they are being closed out at time $t+1$. As such, the net cast outflow, discounted to time $t$ at the risk-adjusted interest rate, arising from the hedging instruments purchased at time $t$ is

$$
\sum_{i=1}^{2} h_{t}^{(i)}\left(H_{t}^{(i)}\left(T_{t}^{(i)}, K_{t}^{(i)}\right)-\left(1+r_{a}\right)^{-1} H_{t+1}^{(i)}\left(T_{t}^{(i)}, K_{t}^{(i)}\right)\right) .
$$

Consequently, the total net cash outflow, discounted to time $t_{0}$ at the risk-adjusted interest rate, arising from all hedging instruments involved in the dynamic hedge is

$$
\sum_{t=t_{0}}^{t_{0}+\omega-x_{0}-1}\left(1+r_{a}\right)^{-\left(t-t_{0}\right)} \sum_{i=1}^{2} h_{t}^{(i)}\left(H_{t}^{(i)}\left(T_{t}^{(i)}, K_{t}^{(i)}\right)-\left(1+r_{a}\right)^{-1} H_{t+1}^{(i)}\left(T_{t}^{(i)}, K_{t}^{(i)}\right)\right) .
$$

We may therefore evaluate the performance of the dynamic hedge using the VaR at the $99.5 \%$ confidence level (the 99.5th percentile) of the distribution of

$$
\mathcal{L}\left(t_{0}, r_{a}\right)+\sum_{t=t_{0}}^{t_{0}+\omega-x_{0}-1}\left(1+r_{a}\right)^{-\left(t-t_{0}\right)} \sum_{i=1}^{2} h_{t}^{(i)}\left(H_{t}^{(i)}\left(T_{t}^{(i)}, K_{t}^{(i)}\right)-\left(1+r_{a}\right)^{-1} H_{t+1}^{(i)}\left(T_{t}^{(i)}, K_{t}^{(i)}\right)\right)
$$

which represents the total discounted cash outflows of the hedged position. The empirical distribution of (14) can be obtained using the following procedure:

1. Calculate the values of $h_{t_{0}}^{(i)}$ and $H_{t_{0}}^{(i)}\left(T_{t_{0}}^{(i)}, K_{t_{0}}^{(i)}\right)$ using the exact formulas presented in Sections 4.1, 4.2, 4.3 and 5.3.

2. Simulate a large number of mortality scenarios, given the information up to and including time $t_{0}$, under the real-world probability measure. For each scenario, perform the following calculations:

(a) Calculate the realized value of $\mathcal{L}\left(t_{0}, r_{a}\right)$.

(b) Calculate the realized values of $H_{t}^{(i)}\left(T_{t}^{(i)}, K_{t}^{(i)}\right)$ for $t=t_{0}+1, \ldots, t_{0}+\omega-x_{0}-1$ and $H_{t+1}^{(i)}\left(T_{t}^{(i)}, K_{t}^{(i)}\right)$ for $t=t_{0}, \ldots, t_{0}+\omega-x_{0}-1$, using the exact formulas presented in Sections 4.1, 4.2 and 4.3. 
(c) Calculate the realized values of $h_{t}^{(i)}$ for $t=t_{0}+1, \ldots, t_{0}+\omega-x_{0}-1$, using the exact formulas presented in Sections 4.1, 4.2 and 4.3 (for the denominator of $h_{t}^{(i)}$ ) and the approximation formula in Section 5.3 (for the numerator of $h_{t}^{(i)}$ ).

3. The previous steps yield a large number of realizations of (14), from which an empirical distribution of (14) can be obtained.

\subsection{Value Hedges}

This sub-section is devoted to value hedges, which focus on the variability of the values of the hedged position at a certain future time point, say $\tau$ years from time $t_{0}$. In the following presentation, we consider particularly the situation when $\tau=1$, which is the most relevant to typical capital requirements.

\subsubsection{The Unhedged Position}

At time $t_{0}+1$, for each of those who are alive, a payment of $\$ 1$ is made and the value of the remaining liability becomes $L_{t_{0}+1}$. There is no payment or financial obligation to those who died between time $t_{0}$ and $t_{0}+1$. Consequently, measured in time- $t_{0}$ dollars, the value of the unhedged position at time $t_{0}+1$ is given by

$$
\left(1+r_{a}\right)^{-1}\left(\mathcal{S}_{x_{0}, t_{0}}(1)+\mathcal{S}_{x_{0}, t_{0}}(1) L_{t_{0}+1}\right)
$$

The VaR at the $99.5 \%$ confidence level (the 99.5th percentile) of the distribution of (15), given information up to and including time $t_{0}$, under the real-world probability measure can be used to as a benchmark for the performance of the value hedges.

\subsubsection{The Hedged Position}

As in a static cash flow hedge, the notional amount of the $i$ th hedging instrument in a (static) value hedge is given by

$$
h^{(i)}=\frac{\Delta_{t_{0}}^{(i, L)}}{\Delta_{t_{0}}^{(i, H)}\left(T^{(i)}, K^{(i)}\right)},
$$

for $i=1,2$ and $H=F, C$, or $P$.

Measured in time- $t_{0}$ dollars, the change in the total value of the two hedging instruments is given by

$$
\sum_{i=1}^{2} h^{(i)}\left(\left(1+r_{a}\right)^{-1} H_{t_{0}+1}^{(i)}\left(T^{(i)}, K^{(i)}\right)-H_{t_{0}}^{(i)}\left(T^{(i)}, K^{(i)}\right)\right) .
$$


As a result, the $t_{0}$-value of the hedged position, measured in time- $t_{0}$ dollars, is

$$
\left(1+r_{a}\right)^{-1} \mathcal{S}_{x_{0}, t_{0}}(1)+\mathcal{S}_{x_{0}, t_{0}}(1) L_{t_{0}+1}-\sum_{i=1}^{2} h^{(i)}\left(\left(1+r_{a}\right)^{-1} H_{t_{0}+1}^{(i)}\left(T^{(i)}, K^{(i)}\right)-H_{t_{0}}^{(i)}\left(T^{(i)}, K^{(i)}\right)\right) .
$$

We may hence assess the performance of the value hedge using the VaR at the $99.5 \%$ (the 99.5th percentile) of the distribution of (17), given the information up to and including $t_{0}$, under the real-world probability measure.

\subsubsection{Generating the Empirical Distributions of (15) and (17)}

The following procedure is used to generate the empirical distributions of (15) and (17), given the information up to and including time $t_{0}$, under the real-world probability measure:

1. Calculate the values of $h^{(i)}$ and $H_{t_{0}}^{(i)}\left(T^{(i)}, K^{(i)}\right)$ using the exact formulas presented in Sections 4.1, 4.2, 4.3 and 5.3.

2. Simulate a large number of mortality scenarios under the real-world probability measure.

For each scenario, perform the following calculations:

(a) Calculate the realized value of $\mathcal{S}_{x_{0}, t_{0}}(1)$.

(b) Calculate the realized values of $H_{t_{0}+1}^{(i)}\left(T^{(i)}, K^{(i)}\right)$ using the exact valuation formulas shown in Sections 4.1, 4.2, 4.3.

(c) Calculate the realized values of $L_{t_{0}+1}$ using the approximation method described in Section 5.2.

3. The previous steps yield a large number of realizations of (15) and (17), from which empirical distributions of (15) and (17) can be obtained. ${ }^{9}$

\section{Illustrations}

\subsection{The Estimated Model}

The illustrations in this section are based on the historical data from the English and Welsh male population, with an age range of 40 to 90 and a calibration window of 1964 to 2013.

We estimate the Cairns-Blake-Dowd model (under the real-world probability measure) to the data set under consideration, using the method of least squares as in the original work of Cairns et al. (2006b). Note that the chosen age range implies $\bar{x}=65$. The estimated values of the two

\footnotetext{
${ }^{9}$ We may further incorporate recalibration (drift change) risk into the empirical distributions by (1) recalibrating the assumed model at time $t_{0}+1$ using the historical data plus the realized mortality experience for year $t_{0}+1$, and then (2) calculating the realized values of $H_{t_{0}+1}^{(i)}\left(T^{(i)}, K^{(i)}\right)$ and $L_{t_{0}+1}$ in the second step using the recalibrated model. We refer readers to Cairns (2013) for a discussion on recalibration risk.
} 

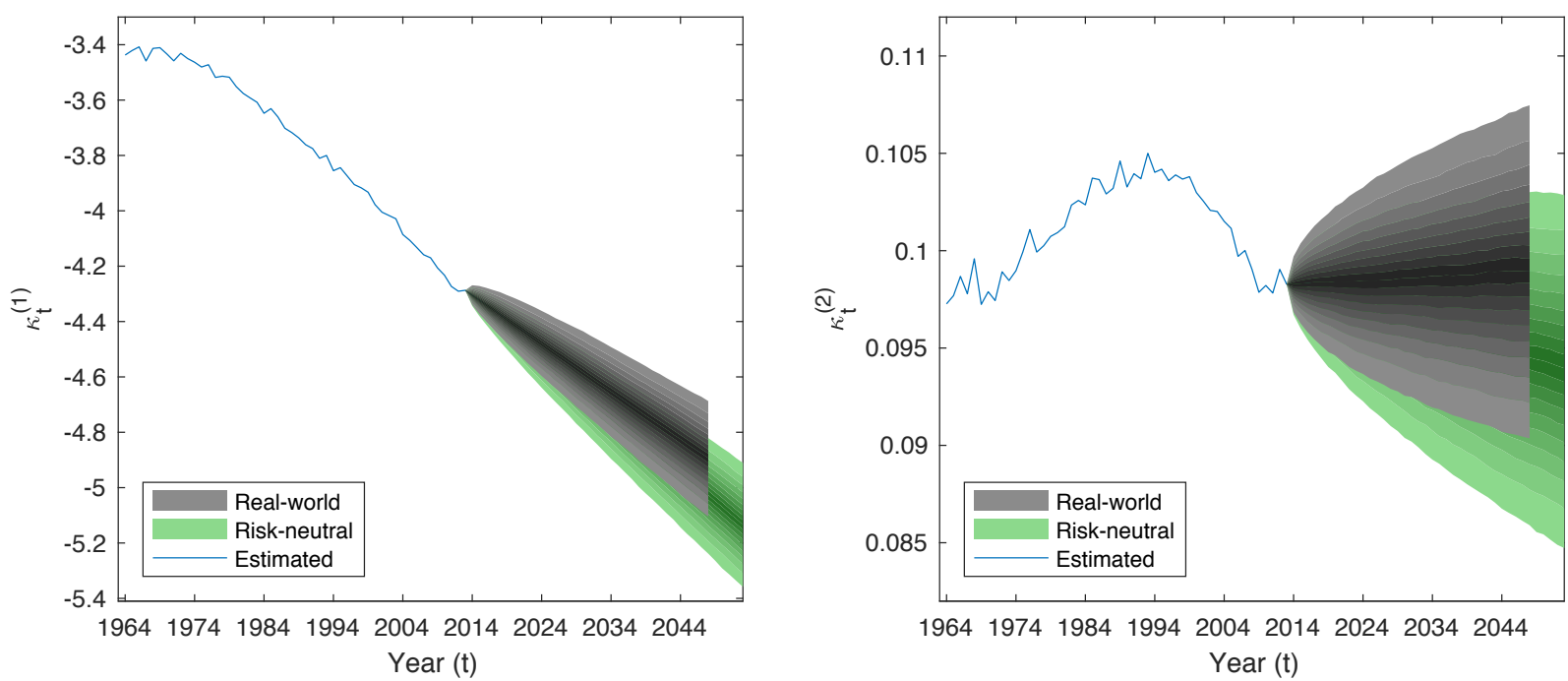

Figure 6: The estimated values of $\kappa_{t}^{(1)}$ and $\kappa_{t}^{(2)}$ for $t=1964, \ldots, 2013$ (the solid lines), and the simulated values of $\kappa_{t}^{(1)}$ and $\kappa_{t}^{(2)}$ for $t>2013$ under the real-world and risk-neutral probability measures (the fan charts).

CBD mortality indexes $\kappa_{t}^{(1)}$ and $\kappa_{t}^{(2)}$ over the calibration window are displayed in 6 (solid lines). A bivariate random walk with drift is then used to capture the dynamics of $\kappa_{t}^{(1)}$ and $\kappa_{t}^{(2)}$. The estimates of the parameters $\boldsymbol{\mu}$ and $\mathbf{A}$ in the process are

$$
\boldsymbol{\mu}=\left(\begin{array}{c}
-1.7346 \times 10^{-2} \\
2.0549 \times 10^{-5}
\end{array}\right) \quad \text { and } \quad \mathbf{A}=\left(\begin{array}{cc}
2.1354 \times 10^{-2} & 4.8559 \times 10^{-4} \\
0 & 7.2807 \times 10^{-4}
\end{array}\right)
$$

respectively.

As mentioned in Section 3, in the baseline calculations we assume that the vector of market prices of risk is $\boldsymbol{\lambda}=(0.175,0.175)^{\prime}$. This assumption implies that under the risk-neutral probability measure, the dynamics of $\kappa_{t}^{(1)}$ and $\kappa_{t}^{(2)}$ follows a bivariate random walk with an adjusted drift vector of $\tilde{\boldsymbol{\mu}}=\left(-2.1167 \times 10^{-2},-1.0686 \times 10^{-4}\right)^{\prime}$. The fan charts in Figure 6 compare the simulated sample paths of $\kappa_{t}^{(1)}$ and $\kappa_{t}^{(2)}$ under the real-world and risk-neutral probability measures.

\subsection{Baseline Results}

The following assumptions are made in our baseline illustrations:

- Three types of index-based hedges are considered: (1) static cash-flow hedges, (2) dynamic cash-flow hedges, and (3) static value hedges (with a horizon of one year).

- All hedges are established at $t_{0}=2013$, the end point of the calibration window.

- The annuity liability is sold to individuals aged $x_{0}=65$ at time $t_{0}=2013$. The limiting age of the annuitants is assumed to be $\omega=100$. 
- For all types of hedges, the composition of the hedge portfolio is either (1) one K-forward (written as a fixed-rate receiver) on each of the two CBD mortality indexes, or (2) one K-put on each of the two CBD mortality indexes. In all hedges, the 'Moneyness' of the two instruments used are identical.

- The time-to-maturity of all hedging instruments (measured at the time when they are purchased) is 15 years.

- Both the CBD mortality indexes and the annuity liability are linked to the mortality experience of the English and Welsh male population.

- The vector of market prices of risk is $\boldsymbol{\lambda}=(0.175,0.175)^{\prime}$.

- The risk-free interest rate is $r_{f}=0.02$ per annum. The corresponding risk-adjusted interest rate $\left(r_{a}\right)$ is calculated using equation (10).

The baseline results for different degrees of 'Moneyness' are shown graphically in Figure $7 .{ }^{10}$ Let us first focus on the static cash flow hedges (the left panel). For the unhedged position, the VaR at the $99.5 \%$ confidence level is 17.44 . Regardless of its 'Moneyness' (i.e., the value of the fixed leg $K$ ), a K-forward hedge can reduce the VaR to a value that is significantly closer to the mean $\left(L_{2013}=16.04\right)$. Also, the VaR it produces reduces (gently) linearly with its fixed leg $K$. This relationship can be explained as follows: When the fixed leg $K$ equals $\mathrm{E}_{t_{0}}^{(Q)}\left[\kappa_{T}^{(i)}\right]$, there is no cash flow exchanges hands at time $t_{0}$ (see Section 4.1), so that the entire hedge cost is payable at maturity when the K-forward is settled. As the fixed leg $K$ is higher than $\mathrm{E}_{t_{0}}^{(Q)}\left[\kappa_{T}^{(i)}\right]$, the amount payable to the counterparty at time $t_{0}$ becomes positive, so that at least part of the cost of the $\mathrm{K}$-forward is payable at time $t_{0}$ instead of at maturity. Because $r_{a}<r_{f}$ (given positive market prices of risk), costs payable at time $t_{0}$ would be lower than that payable at maturity when the time value of money and the interest differential are taken into account, thereby dragging the VaR of the hedged position downwards. Note that when $r_{a}=r_{f}$, the VaR produced by the K-forward hedges would be completely insensitive to the fixed leg $K$ (further illustrations are provided in Section 7.3.1).

The VaR values of the K-forward and K-put (static cash flow) hedges converge as 'Moneyness' becomes very high. This result is expected, because, as explained in Section 4.5, a K-put behaves very similarly to its corresponding K-forward as 'Moneyness' approaches positive infinity. In this particular set-up, the K-put hedges do not outperform the corresponding K-forward hedges for most degrees of 'Moneyness'. To explain, let us consider the following two factors that determine the relative performance between K-put and K-forward hedges:

\section{Cost of hedging}

It is conceivable that a K-put hedge is less costly than the corresponding K-forward hedge, because the hedger needs to pay for only the downside risk when he/she uses a K-put. This

\footnotetext{
${ }^{10}$ 'Moneyness' is defined in equation (8).
} 

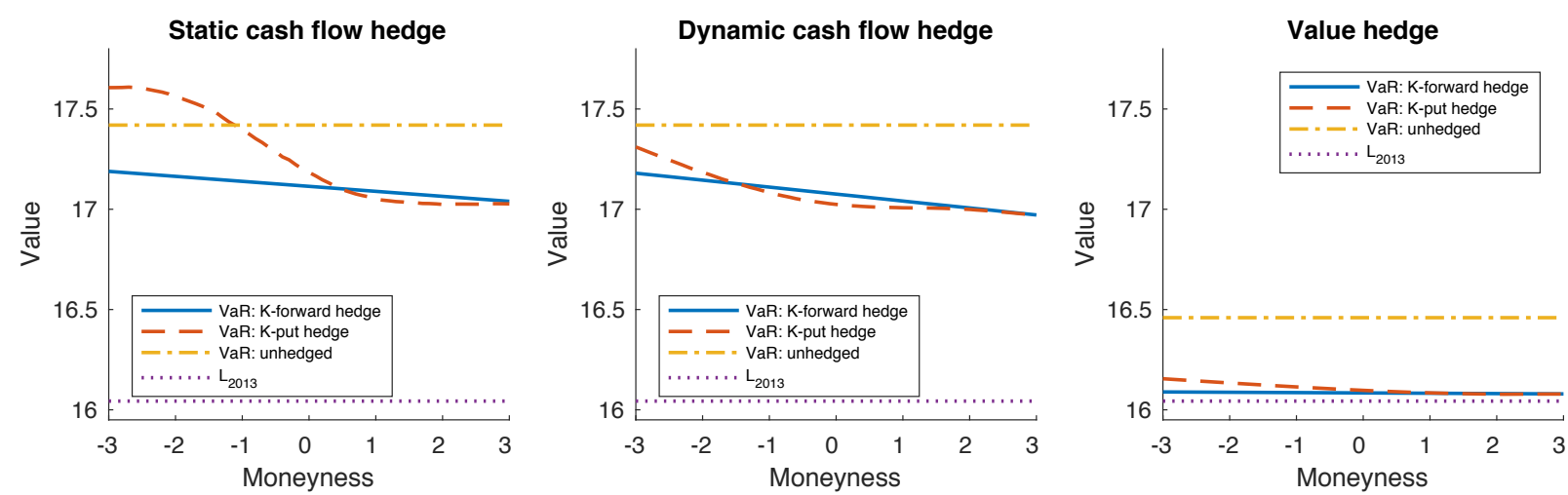

Figure 7: The baseline results of the static cash flow hedges (the left panel), dynamic cash flow hedges (the middle panel) and value hedges (the right panel) at different degrees of 'Moneyness'.
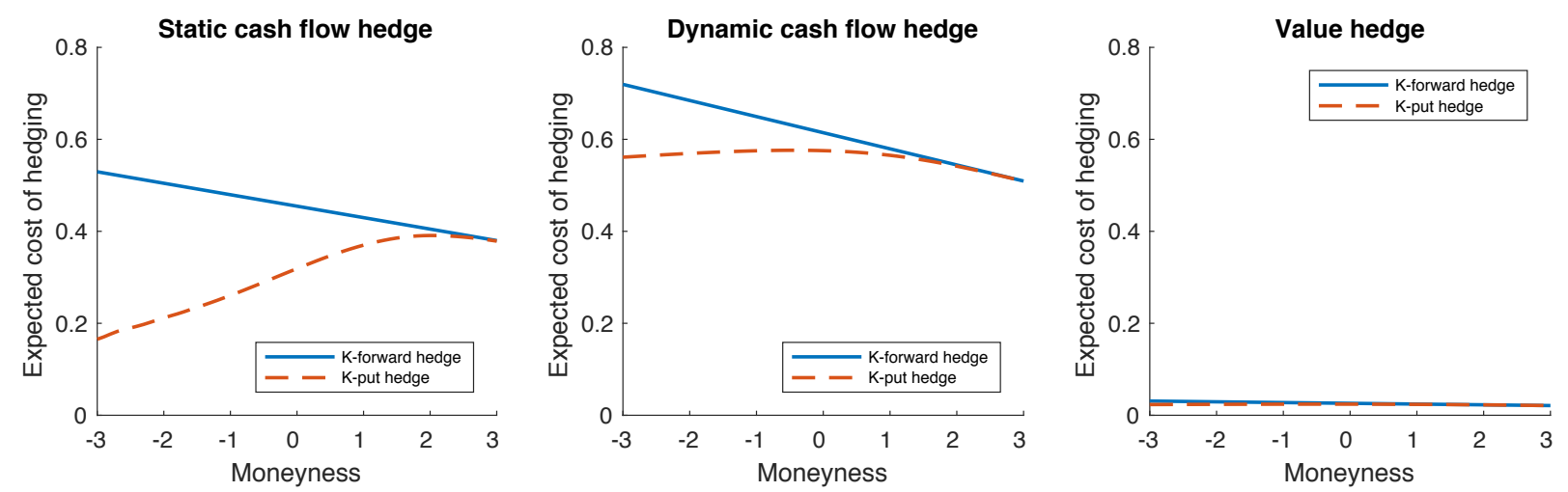

Figure 8: The expected costs of hedging for the baseline static cash flow hedges (the left panel), dynamic cash flow hedges (the middle panel) and value hedges (the right panel) at different degrees of 'Moneyness'.

fact can be observed in the left panel of Figure 8, which compares the expected costs of hedging for the K-put and K-forward static cash flow hedges. ${ }^{11}$

\section{Effectiveness as a hedging instrument}

As demonstrated in the middle panels of Figure 3, the delta of a K-put is always smaller in magnitude than that of the corresponding K-forward. As such, the hedge ratio (the notional amount) of a K-put hedge is always larger in magnitude than that of the corresponding Kforward hedge. Hence, compared to a K-forward hedge, a K-put hedge is more effective in the sense that it will pay a larger payoff to the hedger in an adverse scenario.

To illustrate the interaction between these two offsetting factors, let us analyze the K-forward and K-put static cash flow hedges more deeply. In each panel of Figure 9, 10,000 red dots (for the K-put hedge) and 10,000 blue dots (for the K-forward hedge) are shown. The $x$-coordinate of each

\footnotetext{
${ }^{11}$ For a static cash flow hedge, the expected cost of hedging is calculated as the real-world expected value of (11) given information up to and including time $t_{0}$.
} 

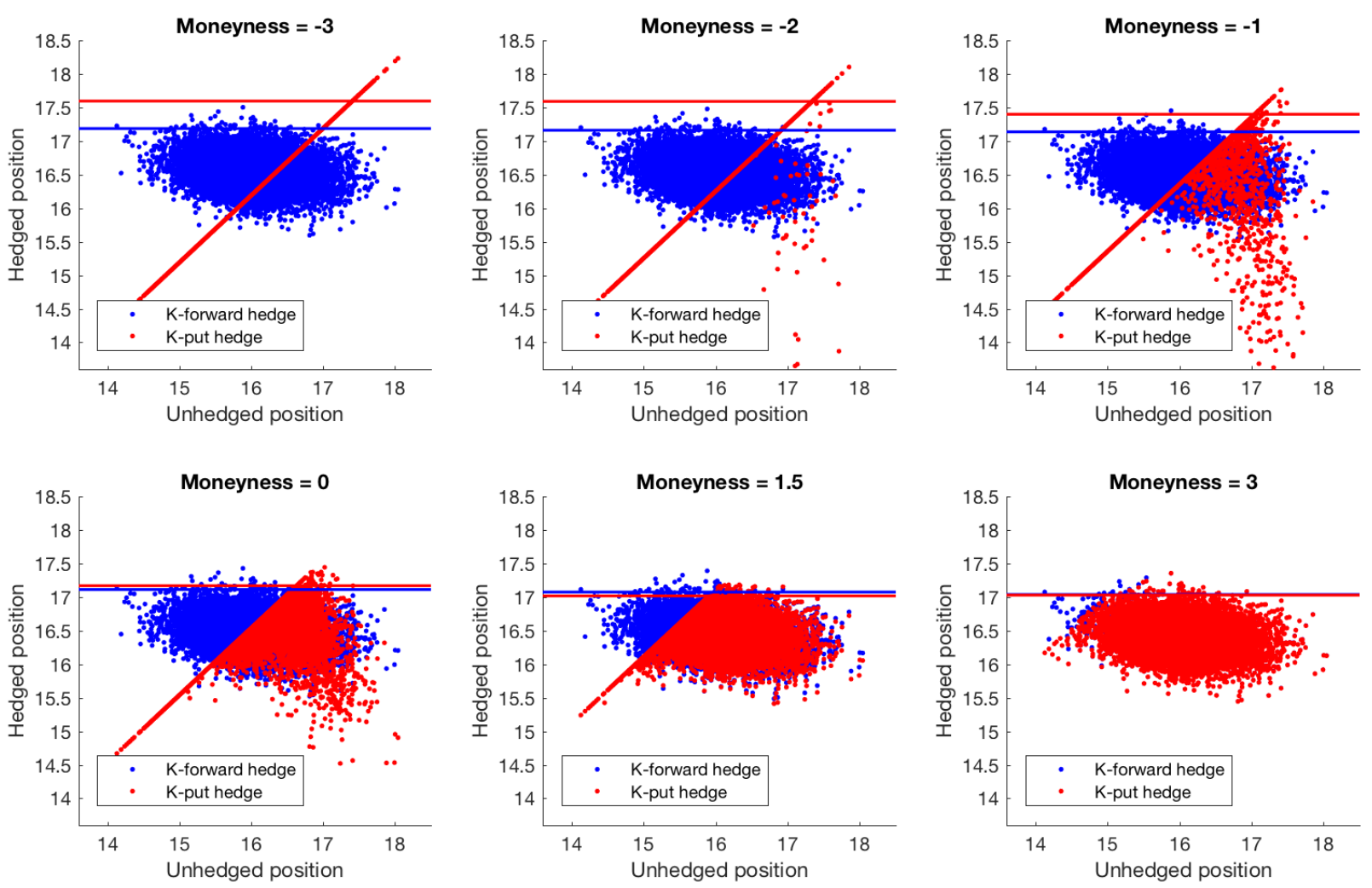

Figure 9: Scatted plots showing the actions of the K-forward and K-put hedges with different degrees of 'Moneyness' in 10,000 simulated mortality scenarios.

dot corresponds to a simulated value of the unhedged position $\mathcal{L}\left(t_{0}, r_{a}\right)$, whereas the $y$-coordinate represents the corresponding simulated value of the hedged position (specified by (12)). The dots shown can be interpreted as follows:

- In a simulated scenario when a hedge takes no action, the dot associated with the scenario and the hedge should lie on a 45 degree line. ${ }^{12}$

- In a simulated scenario when a hedge benefits the hedger, the dot associated with the scenario and the hedge should lie below the 45 degree line, and vice versa.

- The VaR of the hedged position is the vertical position of the 500th dot from top to bottom. The VaR of each hedge is shown as a horizontal solid line in the diagram.

- The clouds of blue dots are symmetric, due to the K-forwards' symmetric payoff structures. The height of each cloud of blue dots is smaller than the width, suggesting that a K-forward hedge reduces the general dispersion of portfolio values. For many of the blue dots on the left, the $y$-coordinate is higher than the $x$-coordinate. This observation suggests that the

\footnotetext{
${ }^{12}$ The 45 degree line may not pass through the origin due to a non-zero hedge cost.
} 
hedger has to sacrifice his/her upside potential when he/she chooses to use a K-forward hedge.

- When 'Moneyness' becomes very negative, all red dots lie on a 45 degree line since the K-puts never yield a payoff. In the other extreme, the clouds of red and blue dots tend to overlap each other, because, as discussed in Section 4.4, a K-put converges to a K-forward as 'Moneyness' becomes very high.

We observe that as 'Moneyness' increases from -3 to a less negative value, the K-put hedge is effective in the sense that it significantly lowers the positions of a good proportion of red dots. However, this effectiveness is not quite manifested in the vertical position of the 500th dot (top to bottom). As such, at those levels of moneyness, the K-put hedge does not yield at lower VaR at the $99.5 \%$ confidence level than the K-forward hedge. When 'Moneyness' equals 1.5, the vertical positions of the highest red lots becomes lower than those of the highest blue dots, so that the K-put hedge results in a lower VaR at the $99.5 \%$ confidence level than the K-forward hedge. We emphasize that the interaction between the two factors depends heavily on the set-up and parameters. In some other circumstances, a K-put hedge outperforms the corresponding K-forward hedge for a much wider range of 'Moneyness'.

When 'Moneyness' is very negative, a static cash flow K-put hedge yields a VaR that is even higher than that of the unhedged position. This outcome arises because when 'Moneyness' is very negative (i.e., the strike value $K$ is very small), the price of a K-put is low but still non-zero, yet none of the simulated CBD mortality indexes falls below the strike value so that the K-put never takes any action in offsetting adverse financial outcomes.

Next, we turn to the dynamic cash flow hedges (the middle panel of Figure 7). The comments made on the static cash flow hedges also apply to the dynamic cash flow hedges. Strikingly, in contrast to what was found in the previous studies by Cairns (2011) and Zhou and Li (2017a), we observe that the dynamic cash flow hedges are only moderately more effective than their static counterpart in terms of reduction in VaR at the $99.5 \%$ confidence level. ${ }^{13}$ This outcome may be attributed to the empirical fact that a dynamic hedge is substantially more costly than a static hedge (see the left and middle panels of Figure 8), and the increase in the cost of hedging offsets the risk reduction benefits arising from the periodic adjustments. ${ }^{14}$

Finally, we consider the (one-year) value hedges (the right panel of Figure 7). Regardless of whether K-puts or K-forwards are used, a value hedge brings the VaR at the $99.5 \%$ confidence level down to a value that is almost equal to the mean $\left(L_{2013}=16.04\right)$. The remarkable reduction in VaR may be attributed to the fact that the one-year value hedge requires the hedger to hold the hedging instruments for only one year, so that the expected cost of a value hedge is significantly

\footnotetext{
${ }^{13}$ Cairns (2011) and Zhou and Li (2017a) did not incorporate costs of hedging into their results.

${ }^{14}$ For a dynamic cash flow hedge, the expected cost of hedging is computed as the real-world expected value of (13) given information up to and including time $t_{0}$.
} 
lower compared to the corresponding cash flow hedges (see the right panel of Figure 8). ${ }^{15}$

\subsection{Sensitivity Tests}

In this sub-section, we examine the sensitivity of the hedging results to (1) changes in the market prices of risk, (2) changes in the times-to-maturity of the hedging instruments, and (3) changes in the risk-free interest rate.

\subsubsection{The Market Prices of Risk}

We now consider three alternative sets of market prices of risk, $(0,0)^{\prime},(0.3,0.3)^{\prime}$ and $(0.5,0.5)^{\prime}$, while retaining all other baseline assumptions. In the extreme case when $\boldsymbol{\lambda}=(0,0)^{\prime}$, the K-put and K-forward hedges are costless.

The results based on the alternative sets of market prices of risk are displayed in Figure 10. When $\boldsymbol{\lambda}=(0,0)^{\prime}$, we have $r_{a}=r_{f}$, and in the absence of the interest rate differential, the result of the K-forward hedge is completely insensitive to its 'Moneyness' (the fixed leg $K$ ). Also, when $\boldsymbol{\lambda}=(0,0)^{\prime}$, all hedges are costless, and therefore the benefit of dynamically adjusting a hedge to the reduction in VaR becomes more apparent.

As the market prices of risk increase, the cost advantage of a K-put hedge over the corresponding K-forward hedge becomes more significant. Consequently, when the market prices of risk are increased from 0.175 (the baseline assumption) to 0.3, a K-put hedge yields a lower VaR than the corresponding K-forward hedge for a wider range of 'Moneyness'. When the market prices of risk are further increased to 0.5, a K-put hedge always results in a lower VaR at the $99.5 \%$ confidence level than the corresponding K-forward hedge; however, at such high market prices of risk, the $\mathrm{VaR}$ of the hedged position (regardless of which instruments are used) is always higher than that of the unhedged position, meaning that a longevity hedge is no longer economically justifiable.

Finally, we observe that for all values of $\boldsymbol{\lambda}$ under consideration, the (one-year) value hedges can always bring the VaR at the $99.5 \%$ confidence level to a value that is very close to the mean liability value. The insensitivity to the market prices of risk may again be attributed to the fact that in such hedges, the hedger needs to hold the hedging instruments for only one year, so that the impact of a change in the market prices of risk is rather modest.

\subsubsection{The Times-to-maturity of the Hedging Instruments}

We now consider three alternative times-to-maturity, 10, 20 and 30 years, while retaining all other baseline assumptions. The results based on the alternative times-to-maturity are shown in Figure 11. Several noteworthy observations can be made.

\footnotetext{
${ }^{15}$ For a value hedge, the expected cost of hedging is calculated as the negative of the real-world expected value of (16) given information up to and including time $t_{0}$.
} 


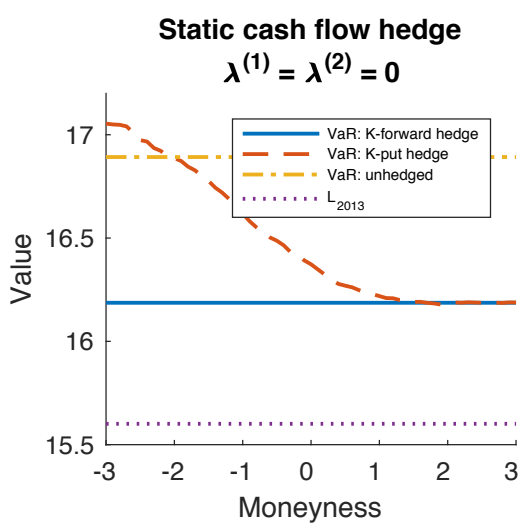

Static cash flow hedge $\lambda^{(1)}=\lambda^{(2)}=0.3$

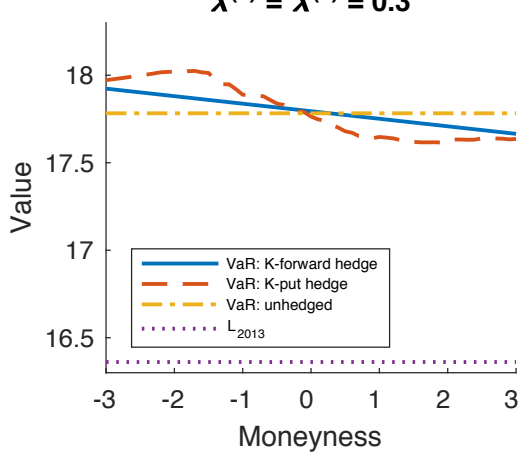

Static cash flow hedge

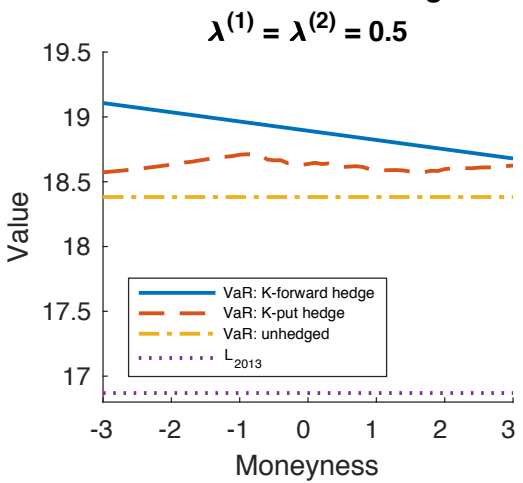

Dynamic cash flow hedge

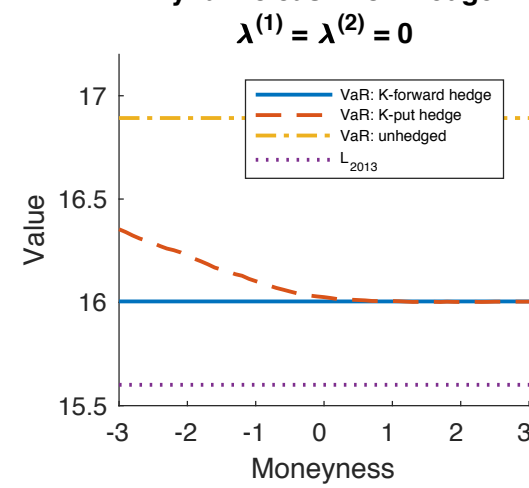

Dynamic cash flow hedge

$\lambda^{(1)}=\lambda^{(2)}=0.3$

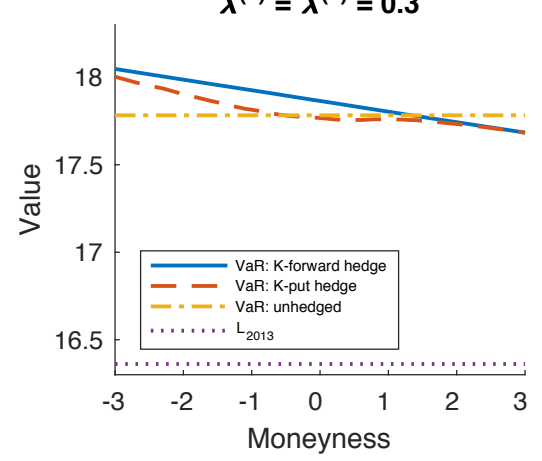

Dynamic cash flow hedge

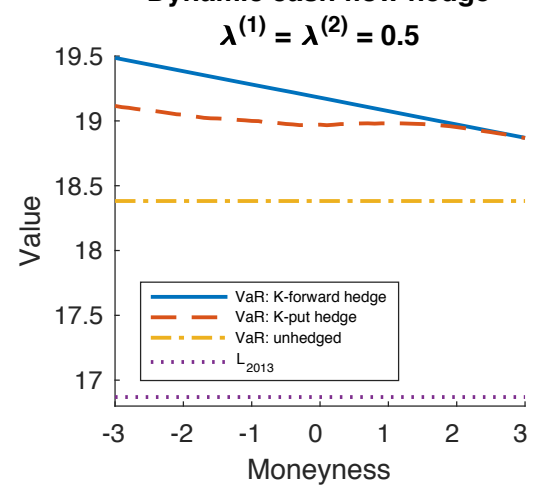

Value hedge

$\lambda^{(1)}=\lambda^{(2)}=0$

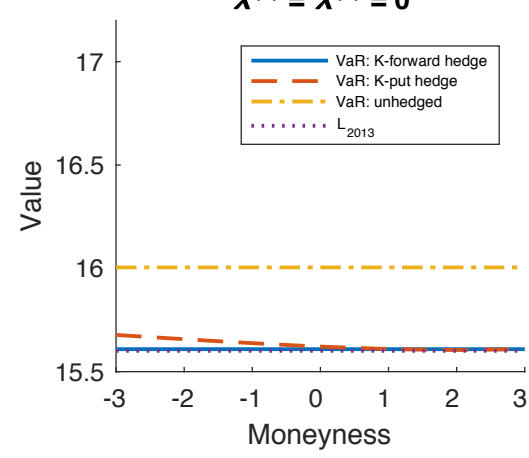

Value hedge

$\lambda^{(1)}=\lambda^{(2)}=0.3$

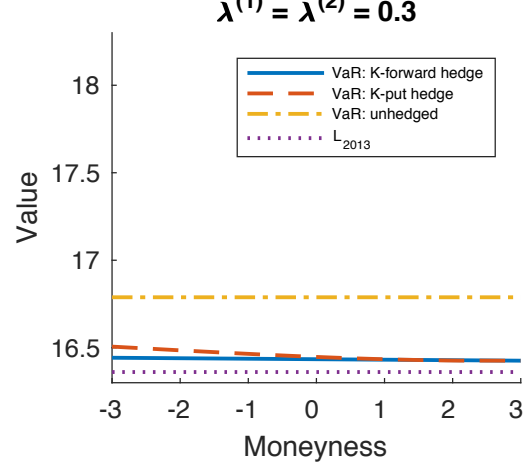

Value hedge

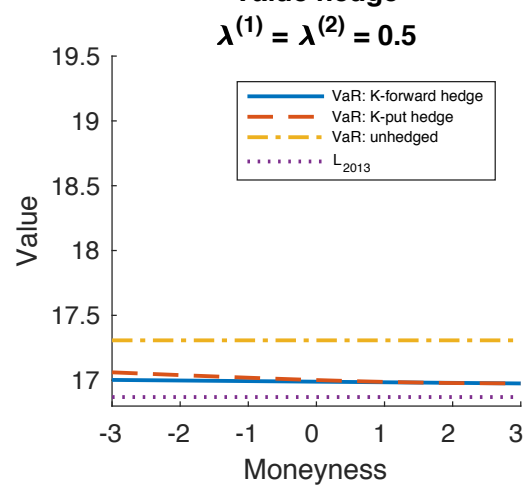

Figure 10: The performance of the static cash flow hedges (the left panel), dynamic cash flow hedges (the middle panel) and value hedges (the right panel) at different degrees of 'Moneyness', when three alternative sets of market prices of risk, $(0,0)^{\prime},(0.3,0.3)^{\prime}$ and $(0.5,0.5)^{\prime}$, are considered. 


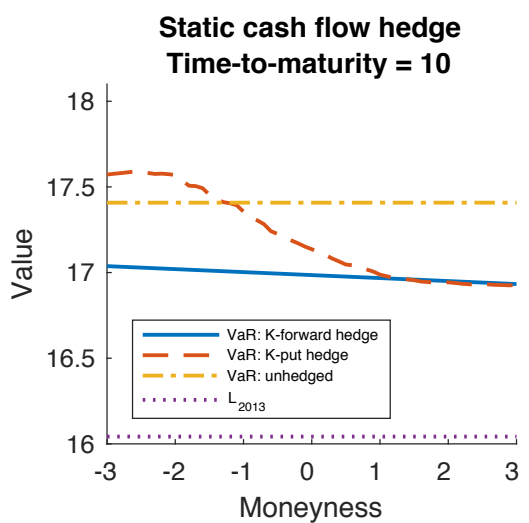

Static cash flow hedge Time-to-maturity $=\mathbf{2 0}$

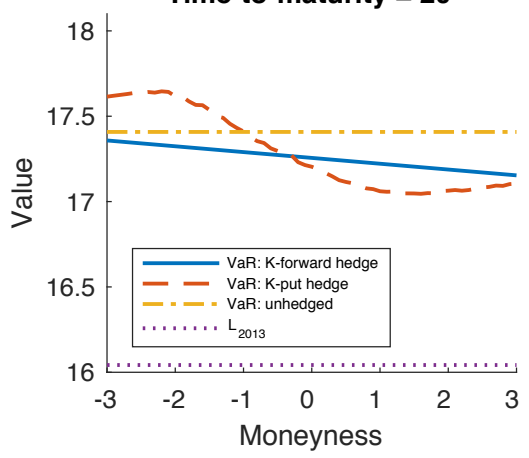

Static cash flow hedge Time-to-maturity $=\mathbf{3 0}$

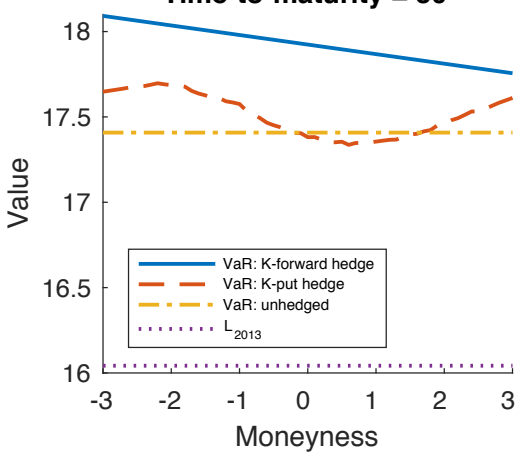

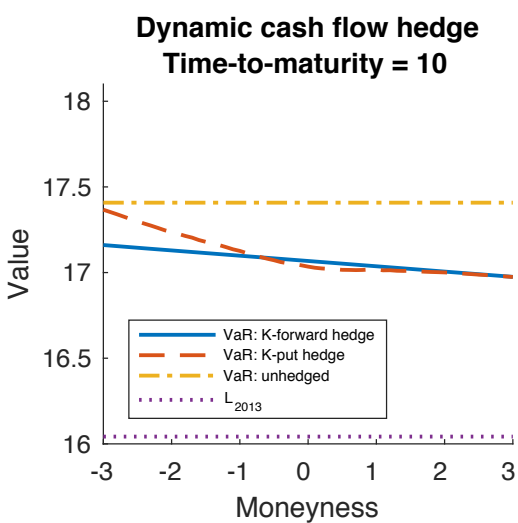

Dynamic cash flow hedge Time-to-maturity $=\mathbf{2 0}$

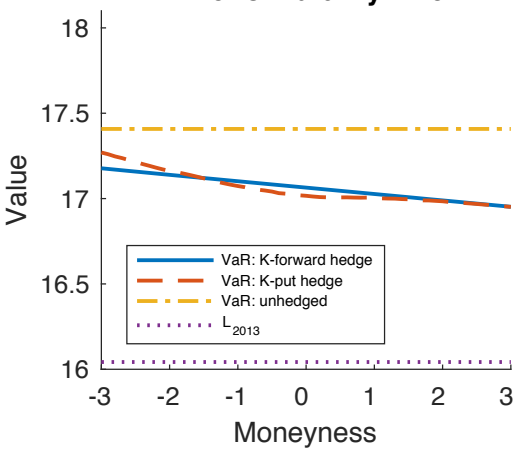

Dynamic cash flow hedge Time-to-maturity $=\mathbf{3 0}$

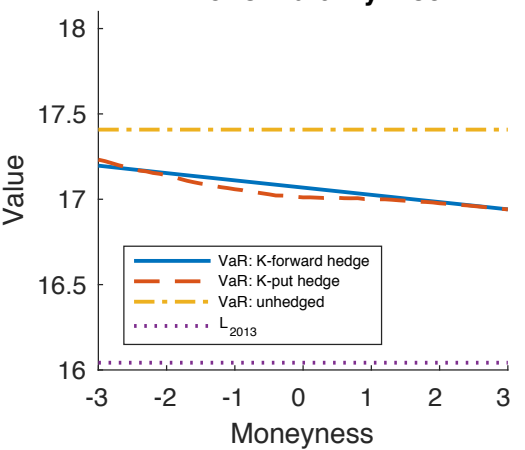

Value hedge Time-to-maturity $=10$
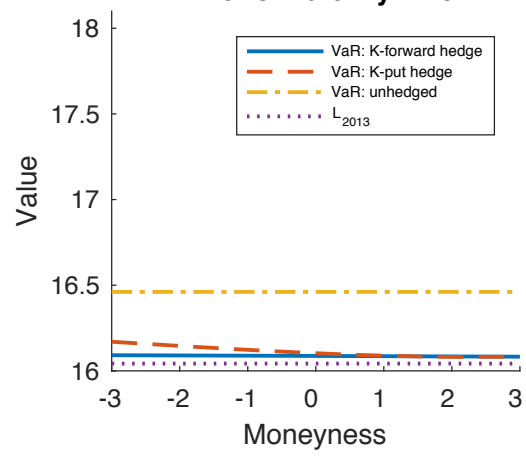

Value hedge

Time-to-maturity $=\mathbf{2 0}$

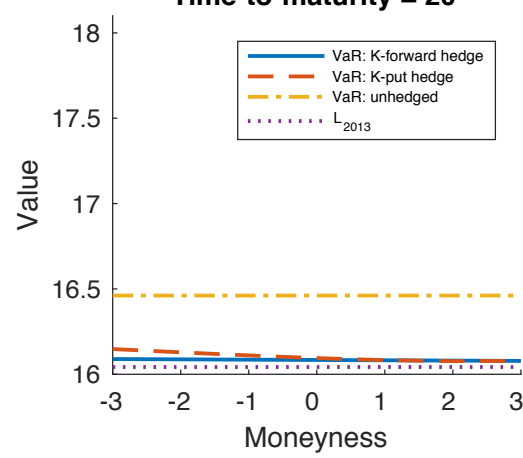

Value hedge

Time-to-maturity $=\mathbf{3 0}$

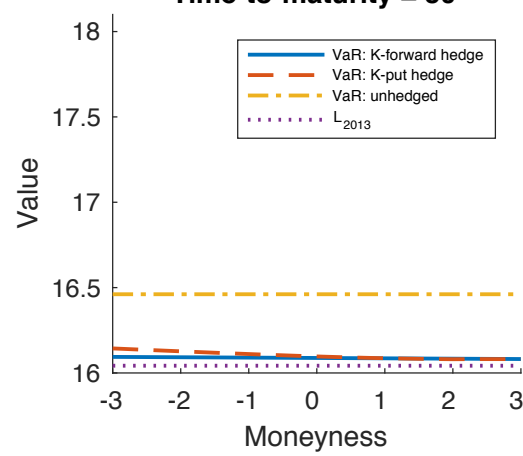

Figure 11: The performance of the static cash flow hedges (the left panel), dynamic cash flow hedges (the middle panel) and value hedges (the right panel) at different degrees of 'Moneyness', when three alternative times-to-maturity, 10, 20 and 30 years, are considered. 
First, for the static cash flow hedges, as the times-to-maturity lengthen, the range of 'Moneyness' over which the red dashed line (representing the VaR produced by the K-put hedge) lies below the solid blue line (representing the VaR produced by the K-forward hedge) becomes wider. This outcome is because when the times-to-maturity rise, all hedging instruments become more costly and hence the cost advantage of K-puts over K-forwards becomes more significant.

Second, for the static cash flow hedges, when the times-to-maturity are increased to 30 years, the K-forward hedge is never economically justifiable and the K-put hedge is economically justifiable for only a very narrow range of 'Moneyness'. This result is due in part to the fact that the hedging instruments are very costly when their times-to-maturity are as high as 30 years, and in part to the fact that a time-to-maturity of 30 years is too long relative to the average time at which the annuity payments are made.

Third, for the dynamic cash flow hedges, the risk reduction is robust to changes in the timesto-maturity of the hedging instruments. This observation is in line with what was found by Zhou and Li (2017a; Online Appendix), who argued that such robustness arises because in an annually adjusted hedge, the hedging instruments acquired at each time point are responsible for hedging the one-year-ahead uncertainty only. For similar reasons, we also observe that the results of the value hedges are robust to changes in the times-to-maturity of the hedging instruments.

\subsubsection{The Risk-free Interest Rate}

We now consider three alternative risk-free interest rates, 0.01, 0.03 and 0.04 , while retaining all other baseline assumptions. Note that according to equation (10), the hedgers' risk-adjusted interest rate changes as we alter the assumed risk-free interest rate.

The results based on the alternative risk-free interest rates are reported in Figure 12. It can be observed that the hedging performance of all three types of hedges is highly robust to changes in the risk-free interest rate. The high robustness can be explained as follows:

- As the risk-free interest rate increases, the longevity deltas of both the annuity liability and hedging instruments become smaller in magnitude. As a consequence, the notional amounts $\left(h^{(i)}\right.$ in static hedges and $h_{t}^{(i)}$ in dynamic hedges), each of which is calculated as the ratio of the longevity deltas of the annuity liability and the relevant hedging instrument, remain fairly unchanged.

- As the risk-free interest rate increases, the risk-adjusted interest rate for discounting realized cash flows also increases. Because the cash flows associated with both the hedged and unhedged positions are discounted more heavily, the net effect tends to be small. 


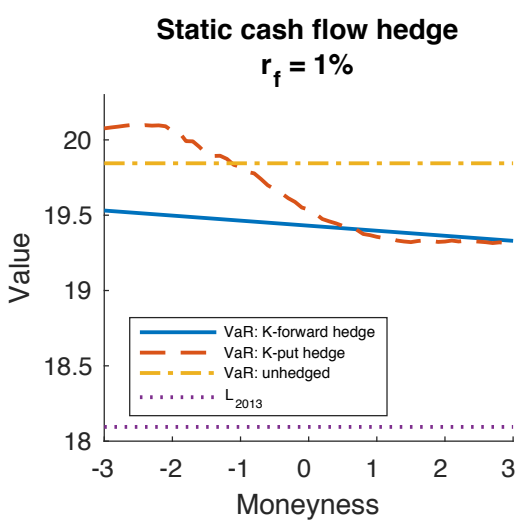

Static cash flow hedge $r_{f}=3 \%$

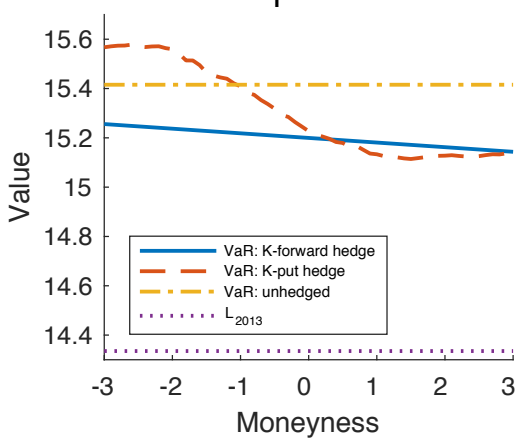

Static cash flow hedge

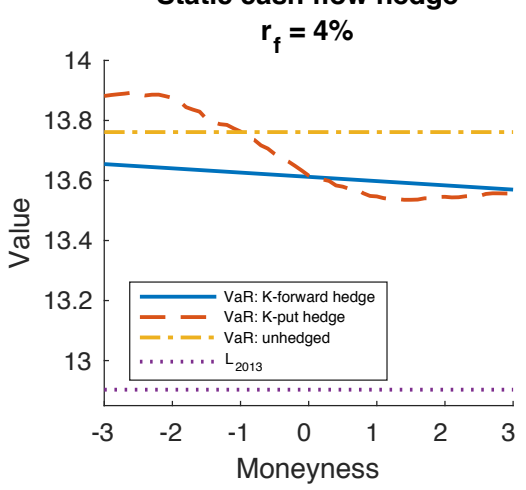

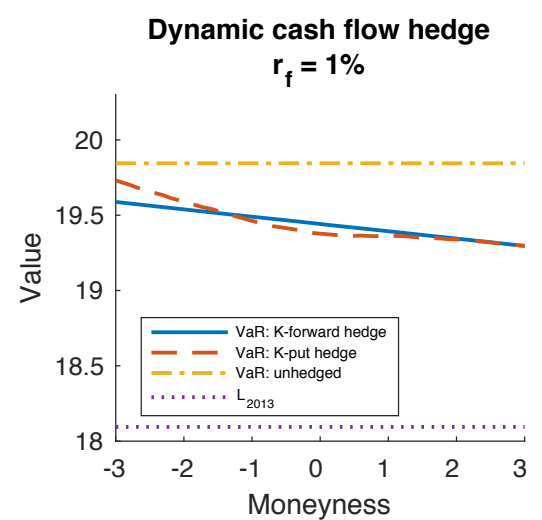

Dynamic cash flow hedge $r_{f}=3 \%$

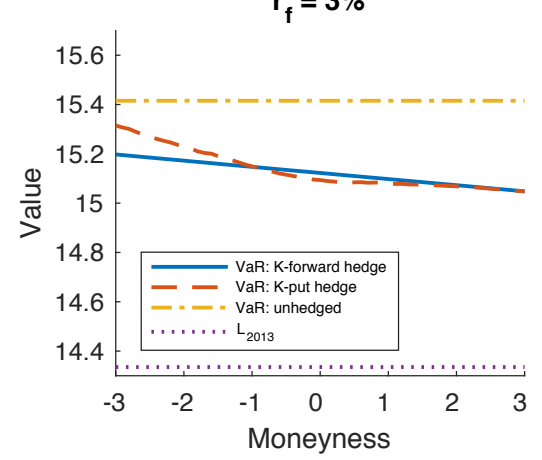

Dynamic cash flow hedge $r_{f}=4 \%$

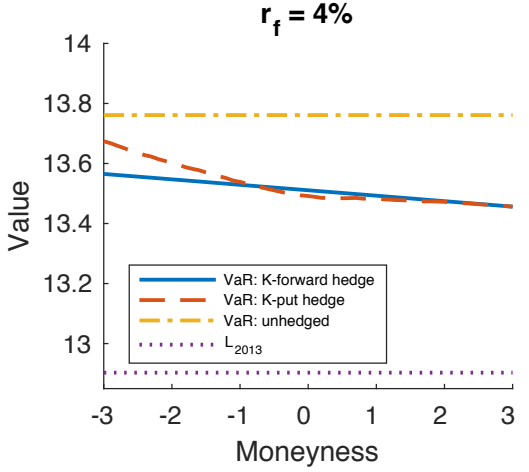

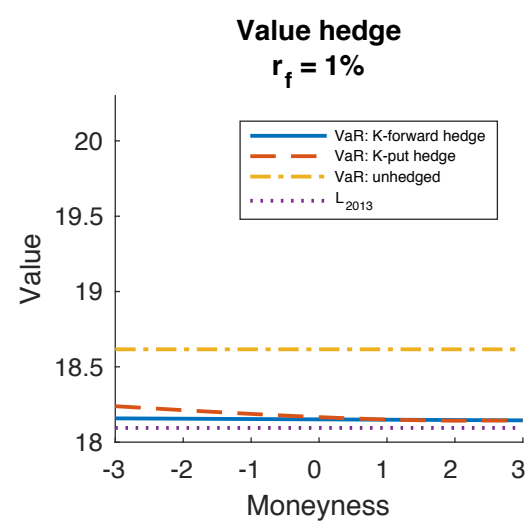

Value hedge $r_{f}=3 \%$

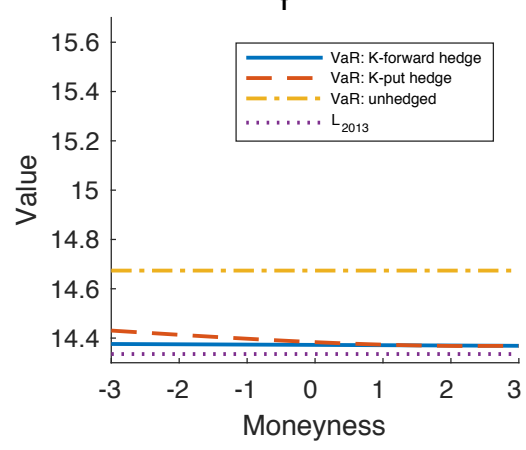

Value hedge $r_{f}=4 \%$

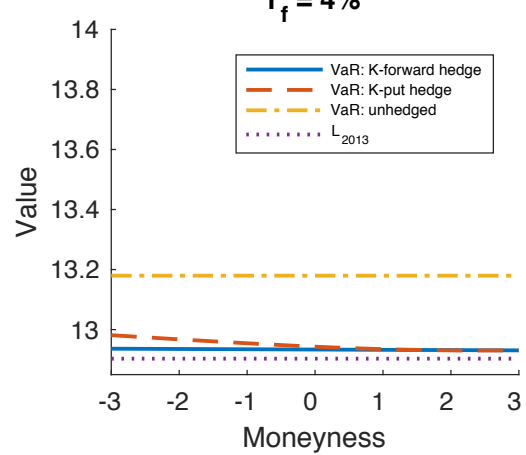

Figure 12: The performance of the static cash flow hedges (the left panel), dynamic cash flow hedges (the middle panel) and value hedges (the right panel) at different degrees of 'Moneyness', when three alternative risk-free interest rates, 0.01, 0.03 and 0.04, are considered. 


\section{Conclusion}

As parametric mortality indexes are rich in information content and interpretable, they are likely to facilitate future development of the market for longevity risk transfers. This paper complements the previous studies on parametric mortality indexes in several aspects.

First, we have introduced K-options written on the CBD mortality indexes (which were found to satisfy the three key criteria for parametric mortality indexes). We have provided explanations as to how K-calls and puts may be utilized by different types of hedgers who may have different exposures to $\mathrm{K} 1$ and $\mathrm{K} 2$ risks. We have also examined the relative performance between K-option hedges and K-forward hedges under different circumstances.

Second, we have derived exact analytical pricing formulas for K-forwards and options, using the statistical and structural properties of the stochastic process in a risk-neutral version of the Cairns-Blake-Dowd model. The analytical pricing formulas not only enable us to more readily estimate the fair values of $\mathrm{K}$-forwards and options in various market conditions, but also spare us from the need for computationally demanding nested simulations in the evaluation of dynamic cash flow hedges and value hedges.

Third, using K-forwards and options as hedging instruments, we have developed three types of longevity hedges: static cash flow hedges, dynamic cash flow hedges, and static value hedges. In all of them, the analytical expressions for the longevity deltas of K-forwards and options (also derived in this paper) play an important role. We illustrate the three types of longevity hedges using real mortality data and previously estimated market prices of risk. Highlights of the empirical work include the following:

- For static/dynamic cash flow hedges, a K-put hedge tends to yield a lower VaR compared to the corresponding K-forward hedge when the market prices of risk are high. However, if the market prices of risk are too high, both K-put and K-forward hedges may no longer be economically justifiable.

- For static cash flow hedges, a K-put hedge tends to result in a smaller VaR compared to the corresponding K-forward hedge when the times-to-maturity of the hedging instruments are long.

- Value hedges are highly effective, even when the market prices of risk are high.

- Dynamic cash flow hedges and value hedges are robust relative to changes in the times-tomaturity of the hedging instruments.

- All three types of hedges are robust relative to changes in the risk-free interest rate.

We acknowledge that European calls and puts are not the only options that can be written on the CBD mortality indexes. Recently, Cairns and Boukfaoui (2017) considered a call spread 
on a hedge index (an index that is related to the liability being hedged), with a payoff function that is specified as follows:

$$
\max \left(0, \min \left(\frac{H I-A P}{E P-A P}, 1\right)\right)
$$

where $H I$ is the hedge index, and $A P$ and $E P$ represent the attachment and exhaustion points of the security, respectively. In future research, it would be interesting to study K-options with a payoff function similar to (18). Such K-options may more easily attract capital market investors, as payoff functions similar to (18) are often seen in catastrophe bonds, which are widely traded in today's market. In addition, we are aware that an annuity liability depends on the trajectories of the CBD mortality indexes over a certain period of time but a European K-option is linked to the value of a CBD mortality index on the maturity date only. This mismatch may have resulted in some structural basis risk, which may be reduced if path-dependent K-options are used instead of European K-options.

We also acknowledge that a bivariate random walk with drift may not be the most appropriate process for the dynamics of the CBD mortality indexes. For instance, using Tiao and Box's (1981) procedure, Chan et al. (2014) found that a VARIMA $(5,1,0)$ model is needed to adequately capture the serial- and cross-correlation in the CBD mortality indexes. ${ }^{16}$ As a matter of fact, the pricing formulas presented in Section 4 are still valid when a VARIMA process is assumed for the CBD mortality indexes instead, provided that $\mathrm{E}_{t}^{(Q)}\left[\kappa_{T}^{(i)}\right]$ and $\operatorname{Var}_{t}^{(Q)}\left[\kappa_{T}^{(i)}\right]$ in the pricing formulas are adapted accordingly. In more detail, suppose that under the real-world probability measure, the CBD mortality indexes follow a $\operatorname{VARIMA}(p, 1,0)$ process

$$
\boldsymbol{\kappa}_{t}-\boldsymbol{\kappa}_{t-1}=\boldsymbol{\mu}+\sum_{i=1}^{p} \boldsymbol{\Phi}_{i}\left(\boldsymbol{\kappa}_{t-i}-\boldsymbol{\kappa}_{t-i-1}\right)+\mathbf{A} \boldsymbol{z}_{t}
$$

where $p$ is the autoregressive order, and $\boldsymbol{\Phi}_{i}, i=1, \ldots, p$, are $2 \times 2$ autoregressive coefficient matrices. ${ }^{17}$ As in Section 3, we assume further that the risk-neutral dynamics of the CBD mortality indexes follow the same process as (19), except that the drift vector is changed to $\tilde{\boldsymbol{\mu}}=\boldsymbol{\mu}-\mathbf{A} \boldsymbol{\lambda}$, where $\boldsymbol{\lambda}$ is the vector of market prices of risk. Under these model assumptions, it can be shown that

$$
\mathrm{E}_{t}^{(Q)}\left[\boldsymbol{\kappa}_{T}\right]=\tilde{\boldsymbol{\mu}}+\mathbf{C}_{1} \mathrm{E}_{t}^{(Q)}\left[\boldsymbol{\kappa}_{T-1}\right]+\ldots+\mathbf{C}_{p+1} \mathrm{E}_{t}^{(Q)}\left[\boldsymbol{\kappa}_{T-(p+1)}\right]
$$

where

$$
\mathbf{C}_{i}= \begin{cases}\mathbf{I}+\boldsymbol{\Phi}_{1}, & i=1 \\ \boldsymbol{\Phi}_{i}-\boldsymbol{\Phi}_{i-1}, & i=2, \ldots, p \\ -\boldsymbol{\Phi}_{p}, & i=p+1 \\ \mathbf{0} & i=p+2, \ldots\end{cases}
$$

\footnotetext{
${ }^{16}$ VARIMA stands for Vector Autoregressive Integrated Moving-Average.

${ }^{17}$ The definitions of $\mathbf{A}, \boldsymbol{\mu}$ and $\boldsymbol{z}_{t}$ are provided in Section 3.
} 
$\mathbf{I}$ is a $2 \times 2$ identity matrix and $\mathbf{0}$ is a $2 \times 2$ zero matrix. Also, we have

$$
\operatorname{Var}_{t}^{(Q)}\left[\boldsymbol{\kappa}_{T}\right]=\sum_{i=1}^{T-t-1} \boldsymbol{\Psi}_{i}\left(\mathbf{A} \mathbf{A}^{\prime}\right) \boldsymbol{\Psi}_{i}
$$

where $\boldsymbol{\Psi}_{i}=\sum_{j=1}^{i} \boldsymbol{\Psi}_{i-j} \mathbf{C}_{j}$ and $\boldsymbol{\Psi}_{0}=\mathbf{I}$. Then, for $i=1,2, \mathrm{E}_{t}^{(Q)}\left[\kappa_{T}^{(i)}\right]$ and $\operatorname{Var}_{t}^{(Q)}\left[\kappa_{T}^{(i)}\right]$ in the pricing formula can be obtained as the $i$ th element in $\mathrm{E}_{t}^{(Q)}\left[\boldsymbol{\kappa}_{T}\right]$ and the $(i, i)$ th element in $\operatorname{Var}_{t}^{(Q)}\left[\boldsymbol{\kappa}_{T}\right]$, respectively. Analytical pricing formulas may also be obtained when the CBD mortality indexes are assumed to follow other processes, but the derivation may be more involved.

As Chan et al. (2014) argued, when developing parametric mortality indexes, we need a model that possesses the new-data-invariant property (a property that ensures that the resulting indexes are tractable). It has been found that the among all of the candidate models considered by Dowd et al. (2010), the Cairns-Blake-Dowd model is the only one that possesses this crucial property. Admittedly, the Cairns-Blake-Dowd model may not provide the best goodness-of-fit to historical data, and consequently, a longevity hedge that is developed from the CBD mortality indexes is subject to some residual risk arising from the age, period and/or cohort effects that the CairnsBlake-Dowd model does not capture. The residual risk may possibly be modeled and incorporated into the evaluation of hedge effectiveness using an age-period-cohort structure that is fitted to the residuals of the estimated Cairns-Blake-Dowd model. This important analysis is left for further research.

\section{Acknowledgments}

The authors gratefully acknowledge financial support from SCOR on the 2017 Longevity Risk Project, from which this paper is extracted. This project has been implemented under the Insurance Risk and Finance Research Centre at Nanyang Business School, Singapore.

\section{References}

Bauer, D., Börger, M., and Ruß, J. (2010). On the pricing of longevity-linked securities. Insurance: Mathematics and Economics 46(1): 139-149.

Bauer, D., Börger, M., Ruß, J., and Zwiesler, H.J. (2008). The volatility of mortality. Asia-Pacific Journal of Risk and Insurance 3(1).

Biffis, E., Lin, Y., and Milidonis, A. (2017). The cross-section of Asia-Pacific mortality dynamics: Implications for longevity risk sharing. Journal of Risk and Insurance 84(S1): 515-532.

Blake, D., Cairns, A. J., and Dowd, K. (2006). Living with mortality: Longevity bonds and other mortality-linked securities. British Actuarial Journal 12(1): 153-197. 
Boyer, M. M., and Stentoft, L. (2013). If we can simulate it, we can insure it: An application to longevity risk management. Insurance: Mathematics and Economics 52(1): 35-45.

Cairns, A.J. (2013). Robust hedging of longevity risk. Journal of Risk and Insurance 80(3): 621-648.

Cairns, A.J. (2011). Modelling and management of longevity risk: Approximations to survivor functions and dynamic hedging. Insurance: Mathematics and Economics 49(3): 438-453.

Cairns, A.J., Blake, D., and Dowd, K. (2008). Modelling and management of mortality risk: A review. Scandinavian Actuarial Journal 2008(2-3): 79-113.

Cairns, A.J., Blake, D., and Dowd, K. (2006a). Pricing death: Frameworks for the valuation and securitization of mortality risk. ASTIN Bulletin 36(1): 79-120.

Cairns, A.J., Blake, D., and Dowd, K. (2006b). A two-factor model for stochastic mortality with parameter uncertainty: Theory and calibration. Journal of Risk and Insurance 73(4): 687-718.

Cairns, A.J.G., and El Boukfaoui, G. (2017) Basis risk in index based longevity hedges: A guide for longevity hedgers. Working paper, Heriot-Watt University.

Chan, W.S., Li, J.S.-H., and Li, J. (2014). The CBD mortality indexes: Modeling and applications. North American Actuarial Journal 18(1): 38-58.

Dahl, M. (2004). Stochastic mortality in life insurance: Market reserves and mortality-linked insurance contracts. Insurance: Mathematics and Economics 35(1): 113-136.

Dowd, K., Cairns, A.J., Blake, D., Coughlan, G.D., Epstein, D., and Khalaf-Allah, M. (2010). Backtesting stochastic mortality models: An ex post evaluation of multiperiod-ahead density forecasts. North American Actuarial Journal 14(3): 281-298.

Hao, X., Liang, C., and Wei, L. (2017). Evaluation of credit value adjustment in K-forward. Insurance: Mathematics and Economics 76(C): 95-103.

Hunt, A., and Blake, D. (2015). Forward mortality rates in discrete time I: Calibration and securities pricing. Pensions Institute Discussion Paper PI-1511.

Li, J., Tickle, L., Tan, C.I., and Li, J.S.-H. (2017). Assessing basis risk for longevity transactions-Phase 2. Research report, the Institute and Faculty of Actuaries and the Life and Longevity Markets Association.

Li, J.S.-H. (2010). Pricing longevity risk with the parametric bootstrap: A maximum entropy approach. Insurance: Mathematics and Economics 47(2): 176-186.

Li, J.S.-H., and Ng, A.C.Y. (2011). Canonical valuation of mortality-linked securities. Journal of Risk and Insurance 78(4): 853-884.

Li, N., and Lee, R. (2005). Coherent mortality forecasts for a group of populations: An extension of the Lee-Carter method. Demography 42(3): 575-594.

Liu, Y., and Li, J.S.-H. (2017). The locally linear Cairns-Blake-Dowd model: A note on delta-nuga dedging of longevity risk. ASTIN Bulletin 47(1): 79-151. 
Michaelson, A., and Mulholland, J. (2014). Strategy for increasing the global capacity for longevity risk transfer: Developing transactions that attract capital markets investors. The Journal of Alternative Investments 17(1): 18-27.

Tan, C.I., Li, J., Li, J.S.-H., and Balasooriya, U. (2014). Parametric mortality indexes: From index construction to hedging strategies. Insurance: Mathematics and Economics 59: 285-299.

Wang, C.W., and Yang, S.S. (2013). Pricing survivor derivatives with cohort mortality dependence under the Lee-Carter framework. Journal of Risk and Insurance 80(4): 1027-1056.

Wei, L. (2017). On bilateral counterparty credit risk in longevity-linked security. M.Sc. thesis, University of Manitoba.

Tiao, G.C. and Box, G.E.P. (1981). Modelling multiple time series with applications. Journal of the American Statistical Association 76: 802-816.

Zhou, K.Q. and Li, J.S.-H. (2017a). Dynamic longevity hedging in the presence of population basis risk: A feasibility analysis from technical and economic perspectives. Journal Risk and Insurance 84: 417-437.

Zhou, K.Q., and Li, J.S.-H. (2017b). Delta-hedging longevity risk under the M7-M5 model: The impact of cohort effect uncertainty and population basis risk. Working paper.

\section{A Derivation of the Pricing Formula and Longevity Greeks for K-Calls}

Here we consider a (European) K-call on the $i$ th CBD mortality index, with issue date $t_{0}$, maturity date $T$ and strike value $K$. Throughout this and the next Appendix, we use $r_{f}$ to represent the risk-free interest rate, $I_{A}$ to denote an indicator function which equals 1 if event $A$ occurs and 0 otherwise, and the following shorthand notation:

$$
B=\frac{K-\mathrm{E}_{t}^{(Q)}\left[\kappa_{T}^{(i)}\right]}{\sqrt{\operatorname{Var}_{t}^{(Q)}\left[\kappa_{T}^{(i)}\right]}}
$$

We also use the fact that

$$
\frac{\partial B}{\partial \kappa_{t}^{(i)}}=\frac{-1}{\sqrt{\operatorname{Var}_{t}^{(Q)}\left[\kappa_{T}^{(i)}\right]}}, \quad t<T,
$$

which follows from equations (5) and (6) in the main text. 


\section{A.1 Pricing Formula}

The price of the K-call at time $t$ for $t_{0} \leq t<T$ is given by

$$
\begin{aligned}
C_{t}^{(i)}(T, K) & =\mathrm{E}_{t}^{(Q)}\left[\left(1+r_{f}\right)^{-(T-t)} \max \left(\kappa_{T}^{(i)}-K, 0\right)\right] \\
& =\left(1+r_{f}\right)^{-(T-t)} \mathrm{E}_{t}^{(Q)}\left[\left(\kappa_{T}^{(i)}-K\right) I_{\left\{\kappa_{T}^{(i)}>K\right\}}\right] \\
& =\left(1+r_{f}\right)^{-(T-t)}\left(\mathrm{E}_{t}^{(Q)}\left[\kappa_{t}^{(i)} I_{\left\{\kappa_{T}^{(i)}>K\right\}}\right]-K \mathrm{E}_{t}^{(Q)}\left[I_{\left\{\kappa_{T}^{(i)}>K\right\}}\right]\right) \\
& =\left(1+r_{f}\right)^{-(T-t)}\left(\mathrm{E}_{t}^{(Q)}\left[\kappa_{t}^{(i)} I_{\left\{\kappa_{T}^{(i)}>K\right\}}\right]-K(1-\Phi(B))\right) .
\end{aligned}
$$

Aside, we have

$$
\begin{aligned}
\mathrm{E}_{t}^{(Q)}\left[\kappa_{T}^{(i)} I_{\left\{\kappa_{T}^{(i)}>K\right\}}\right] & =\int_{K}^{+\infty} x \frac{1}{\sqrt{2 \pi \operatorname{Var}_{t}^{(Q)}\left[\kappa_{T}^{(i)}\right]}} \exp \left(-\frac{\left(x-\mathrm{E}_{t}^{(Q)}\left[\kappa_{T}^{(i)}\right]\right)^{2}}{2 \operatorname{Var}_{t}^{(Q)}\left[\kappa_{T}^{(i)}\right]}\right) d x \\
& =\int_{B}^{+\infty}\left(\mathrm{E}_{t}^{(Q)}\left[\kappa_{T}^{(i)}\right]+u \sqrt{\operatorname{Var}_{t}^{(Q)}\left[\kappa_{T}^{(i)}\right]}\right) \frac{1}{\sqrt{2 \pi}} \exp \left(-\frac{1}{2} u^{2}\right) d u \\
& =\mathrm{E}_{t}^{(Q)}\left[\kappa_{T}^{(i)}\right](1-\Phi(B))-\sqrt{\operatorname{Var}_{t}^{(Q)}\left[\kappa_{T}^{(i)}\right]} \int_{B}^{+\infty} \frac{1}{\sqrt{2 \pi}} \exp \left(-\frac{1}{2} u^{2}\right) d\left(-\frac{1}{2} u^{2}\right) \\
& =\mathrm{E}_{t}^{(Q)}\left[\kappa_{T}^{(i)}\right](1-\Phi(B))+\sqrt{\operatorname{Var}_{t}^{(Q)}\left[\kappa_{T}^{(i)}\right]} \phi(B) .
\end{aligned}
$$

It follows that

$$
C_{t}^{(i)}(T, K)=\left(1+r_{f}\right)^{-(T-t)} \sqrt{\operatorname{Var}_{t}^{(Q)}\left[\kappa_{T}^{(i)}\right]}(\phi(B)-B(1-\Phi(B))) .
$$

\section{A.2 Longevity Delta}

For $t_{0} \leq t<T$, the time- $t$ value of the longevity delta for the K-call is given by

$$
\begin{aligned}
\Delta_{t}^{(i, C)}(T, K) & =\frac{\partial C_{t}^{(i)}(T, K)}{\partial \kappa_{t}^{(i)}} \\
& =\frac{\partial}{\partial \kappa_{t}^{(i)}}\left(1+r_{f}\right)^{-(T-t)} \sqrt{\operatorname{Var}_{t}^{(Q)}\left[\kappa_{T}^{(i)}\right]}(\phi(B)-B(1-\Phi(B))) \\
& =\left(1+r_{f}\right)^{-(T-t)} \sqrt{\operatorname{Var}_{t}^{(Q)}\left[\kappa_{T}^{(i)}\right]}\left(\frac{\partial \phi(B)}{\partial \kappa_{t}^{(i)}}-\frac{\partial B}{\partial \kappa_{t}^{(i)}}+\frac{\partial B \Phi(B)}{\partial \kappa_{t}^{(i)}}\right) \\
& =\left(1+r_{f}\right)^{-(T-t)} \sqrt{\operatorname{Var}_{t}^{(Q)}\left[\kappa_{T}^{(i)}\right]}\left(\frac{\partial \phi(B)}{\partial \kappa_{t}^{(i)}}-\frac{\partial B}{\partial \kappa_{t}^{(i)}}+\frac{\partial B}{\partial \kappa_{t}^{(i)}} \Phi(B)+\frac{\partial \Phi(B)}{\partial \kappa_{t}^{(i)}} B\right) \\
& =\left(1+r_{f}\right)^{-(T-t)} \sqrt{\operatorname{Var}_{t}^{(Q)}\left[\kappa_{T}^{(i)}\right]}\left(\frac{B \phi(B)-(-1)+(-\Phi(B))+(-B \phi(B))}{\sqrt{\operatorname{Var}_{t}^{(Q)}\left[\kappa_{T}^{(i)}\right]}}\right) \\
& =\left(1+r_{f}\right)^{-(T-t)}(1-\Phi(B)) .
\end{aligned}
$$




\section{A.3 Longevity Gamma}

For $t_{0} \leq t<T$, the time- $t$ value of the longevity gamma for the K-call is given by

$$
\begin{aligned}
\Gamma_{t}^{(i, C)}(T, K) & =\frac{\partial}{\partial \kappa_{t}^{(i)}} \Delta_{t}^{(i, C)}(T, K) \\
& =-\left(1+r_{f}\right)^{-(T-t)} \frac{\partial}{\partial \kappa_{t}^{(i)}} \Phi(B) \\
& =\left(1+r_{f}\right)^{-(T-t)} \frac{1}{\sqrt{\operatorname{Var}_{t}^{(Q)}\left[\kappa_{T}^{(i)}\right]}} \phi(B) .
\end{aligned}
$$

\section{B Derivation of the Pricing Formula and Longevity Greeks for K-Puts}

Here we consider a (European) K-put on the $i$ th CBD mortality index, with issue date $t_{0}$, maturity date $T$ and strike value $K$.

\section{B.1 Pricing Formula}

The price of the K-put at time $t$ for $t_{0} \leq t<T$ is given by

$$
\begin{aligned}
P_{t}^{(i)}(T, K) & =\mathrm{E}_{t}^{(Q)}\left[\left(1+r_{f}\right)^{-(T-t)} \max \left(K-\kappa_{T}^{(i)}, 0\right)\right] \\
& =\left(1+r_{f}\right)^{-(T-t)} \mathrm{E}_{t}^{(Q)}\left[\left(K-\kappa_{T}^{(i)}\right) I_{\left\{\kappa_{T}^{(i)}<K\right\}}\right] \\
& =\left(1+r_{f}\right)^{-(T-t)}\left(K \mathrm{E}_{t}^{(Q)}\left[I_{\left\{\kappa_{T}^{(i)}<K\right\}}\right]-\mathrm{E}_{t}^{(Q)}\left[\kappa_{T}^{(i)} I_{\left\{\kappa_{T}^{(i)}<K\right\}}\right]\right) \\
& =\left(1+r_{f}\right)^{-(T-t)}\left(K \Phi(B)-\mathrm{E}_{t}^{(Q)}\left[\kappa_{T}^{(i)} I_{\left\{\kappa_{T}^{(i)}<K\right\}}\right]\right) .
\end{aligned}
$$

Aside, using the results from Appendix A.1, we have

$$
\begin{aligned}
\mathrm{E}_{t}^{(Q)}\left[\kappa_{T}^{(i)} I_{\left\{\kappa_{T}^{(i)}<K\right\}}\right] & =\mathrm{E}_{t}^{(Q)}\left[\kappa_{T}^{(i)}\right]-\mathrm{E}_{t}^{(Q)}\left[\kappa_{T}^{(i)} I_{\left\{\kappa_{T}^{(i)}>K\right\}}\right] \\
& =\mathrm{E}_{t}^{(Q)}\left[\kappa_{T}^{(i)}\right]-\mathrm{E}_{t}^{(Q)}\left[\kappa_{T}^{(i)}\right](1-\Phi(B))-\sqrt{\operatorname{Var}_{t}^{(Q)}\left[\kappa_{T}^{(i)}\right]} \phi(B) \\
& =\mathrm{E}_{t}^{(Q)}\left[\kappa_{T}^{(i)}\right] \Phi(B)-\sqrt{\operatorname{Var}_{t}^{(Q)}\left[\kappa_{T}^{(i)}\right]} \phi(B) .
\end{aligned}
$$

It follows that

$$
\left.P_{t}^{(i)}(T, K)=\left(1+r_{f}\right)^{-(T-t)}\left(\sqrt{\operatorname{Var}_{t}^{(Q)}\left[\kappa_{T}^{(i)}\right.}\right] \phi(B)+\left(K-\mathrm{E}_{t}^{(Q)}\left[\kappa_{T}^{(i)}\right]\right) \Phi(B)\right) .
$$




\section{B.2 Longevity Delta}

For $t_{0} \leq t<T$, the time- $t$ value of the longevity delta for the K-put is given by

$$
\begin{aligned}
\Delta_{t}^{(i, P)}(T, K) & =\frac{\partial P_{t}^{(i)}(T, K)}{\partial \kappa_{t}^{(i)}} \\
& =\left(1+r_{f}\right)^{-(T-t)} \sqrt{\operatorname{Var}_{t}^{(Q)}\left[\kappa_{T}^{(i)}\right]}\left(\frac{\partial \phi(B)}{\partial \kappa_{t}^{(i)}}+\frac{\partial B \Phi(B)}{\partial \kappa_{t}^{(i)}}\right) \\
& =\left(1+r_{f}\right)^{-(T-t)} \sqrt{\operatorname{Var}_{t}^{(Q)}\left[\kappa_{T}^{(i)}\right]}\left(\frac{\partial \phi(B)}{\partial \kappa_{t}^{(i)}}+\frac{\partial B}{\partial \kappa_{t}^{(i)}} \Phi(B)+\frac{\partial \Phi(B)}{\partial \kappa_{t}^{(i)}} B\right) \\
& =\left(1+r_{f}\right)^{-(T-t)} \sqrt{\operatorname{Var}_{t}^{(Q)}\left[\kappa_{T}^{(i)}\right]}\left(\frac{B \phi(B)+(-\Phi(B))+(-B \phi(B))}{\sqrt{\operatorname{Var}_{t}^{(Q)}\left[\kappa_{T}^{(i)}\right]}}\right) \\
& =-\left(1+r_{f}\right)^{-(T-t)} \Phi(B) .
\end{aligned}
$$

\section{B.3 Longevity Gamma}

For $t_{0} \leq t<T$, the time- $t$ value of the longevity gamma for the K-put is given by

$$
\begin{aligned}
\Gamma_{t}^{(i, P)}(T, K) & =\frac{\partial}{\partial \kappa_{t}^{(i)}} \Delta_{t}^{(i, P)}(T, K) \\
& =-\left(1+r_{f}\right)^{-(T-t)} \frac{\partial}{\partial \kappa_{t}^{(i)}} \Phi(B) \\
& =\left(1+r_{f}\right)^{-(T-t)} \frac{1}{\sqrt{\operatorname{Var}_{t}^{(Q)}\left[\kappa_{T}^{(i)}\right]}} \phi(B) .
\end{aligned}
$$

